TRABAJOS ORIGINALES

\title{
Lista anotada de árboles y afines en los bosques montanos del sureste peruano: la importancia de seguir recolectando
}

\section{An annotated checklist of trees and relatives in tropical montane forests from southeast Peru: the importance of continue collecting}

\section{William Farfan-Rios ${ }^{1,2 *}$, Karina Garcia-Cabrera ${ }^{1,2}$, Norma Salinas ${ }^{2,3,4}$, Mireya N. Raurau-Quisiyupanqui ${ }^{2}$ y Miles R. Silman ${ }^{1,5}$}

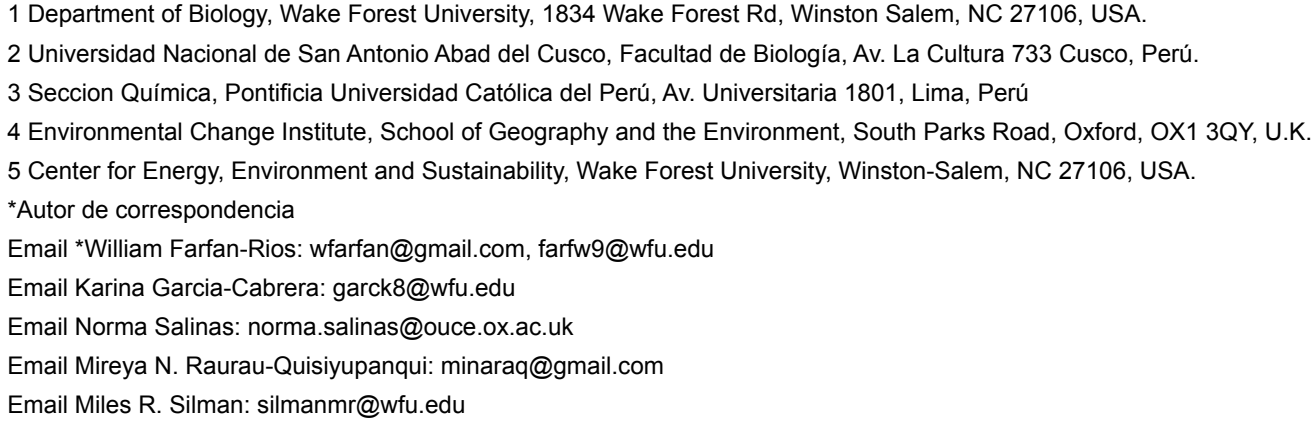

\begin{abstract}
Resumen
Los Andes están considerados como los puntos calientes más diversos de los trópicos, dentro de estos se encuentra el Parque Nacional del Manu, cuyas complejas condiciones climáticas y fisiográficas albergan una mega-diversidad y endemismo. En el presente trabajo se presenta una lista anotada de especies arbóreas y afines a lo largo de un gradiente de elevación desde los bosques submontanos a $800 \mathrm{~m}$ hasta la línea de bosque a $3625 \mathrm{~m}$ en la Reserva de Biosfera del Manu. En base a una red de 21 parcelas permanentes de una hectárea y exploraciones botánicas se sistematiza la información florística por rangos de elevación, distribución geográfica y endemismo. Estos resultados preliminares se traducen en 1108 especies de las cuales el $39.7 \%$ son morfoespecies, el $43 \%$ de las especies determinadas son registros nuevos para la región del Cusco, 15 especies son nuevos registros para la flora peruana, 40 especies son endémicas para Perú y 30 son potenciales especies nuevas para la ciencia. Adicionalmente, se resalta la expansión del rango altitudinal para el $45.2 \%$ de las especies determinadas (302 especies). Estos resultados son una muestra de la alta diversidad arbórea y afines en estos ecosistemas montañosos registrados en tan solo $\sim 20 \mathrm{~km}$ de distancia geográfica, además muestra lo escasamente colectados y poco estudiados que se encuentran. Mas colecciones botánicas son necesarias - estos estudios básicos de florística son imperativos para un mejor entendimiento de la distribución de especies y la función del ecosistemas, además ayudará a responder una de las grandes preguntas en la ecología global moderna, ¿Cómo responderán los bosques tropicales al cambio climático global?
\end{abstract}

Palabras clave: Andes; Bosque montano tropical; cambio climático; diversidad arbórea; distribución de especies; rangos de elevación.

Citación:

Farfan-Rios W., K. Garcia-Cabrera, N. Salinas, M.N. Raurau-Quisiyupanqui \& M.R. Silman. 2015. Lista anotada de árboles y afines en los bosques montanos del sureste peruano: es importante seguir recolectando. Revista peruana de biología 22(2): 145 - 174 (Octubre 2015). doi: http://dx.doi.org/10.15381/rpb.v22i2.11351

Fuentes de financiamiento: Este trabajo fue financiado por: "The United States National Science Foundation", la fundación "Gordon and Betty Moore - Andes to Amazon Program" y "Amazon Conservation Association".

$\begin{array}{ll}\text { Presentado: } & 17 / 11 / 2014 \\ \text { Aceptado: } & 20 / 03 / 2015\end{array}$

$\begin{array}{ll}\text { Aceptado: } & 20 / 03 / 2015 \\ \text { Publicado online: } 14 / 10 / 2015\end{array}$
Información sobre los autores:

Los autores declaran que participaron en: WF: En la compilación, identificación, validación de especies registradas, revisión y redacción del manuscrito; KG: Identificación de especies registradas, revisión y redacción del manuscrito; NS: Identificación de especies registradas, revisión y redacción del manuscrito; MN: Identificación y validación de especies registradas; MS: Identificación de especies registradas y redacción del manuscrito.

Los autores no incurren en conflictos de intereses. 


\begin{abstract}
The tropical Andes and adjacent Amazon are Earth's highest biodiversity hotspot. Manu National Park in southeastern Peru encompasses an entire watershed, ranging from Andean highlands to Amazonian lowlands, and is a megadiverse landscape on the Andes to Amazon transition. Here we present an annotated checklist of trees and related species is along an elevation gradient in the Manu Biosphere Reserve that runs from sub-montane forests at $800 \mathrm{~m}$ elevation up to the tree line at $3625 \mathrm{~m}$. Based on a network of 21 1-hectare permanent tree plots and botanical explorations, the floristic information is systematized by elevation ranges, geographical distribution and endemism. These preliminary results show 1108 species. Of these, $43 \%$ are new records for the region of Cusco, 15 species are new records for the Peruvian flora, 40 species are endemics for Peru, and 30 are potential new species for science. Another $39.7 \%$ are identified to genus or family level and remain morphospecies. Additionally, we show altitudinal range expansion for $45.2 \%$ of identified species (302 species). These results were found in a transect of plots spanning only $20 \mathrm{~km}$ of geographic distance, and are a sample of the high tree diversity in these mountainous ecosystems. The data show how poorly collected and understudied these ecosystems are. Basic floristic studies and collections are imperative for a better understanding of species distribution and function of ecosystems, and the basic biodiversity of the tropical Andes. They will also help to answer a major, unresolved question in modern global ecology of how tropical forests will respond to global climate change.
\end{abstract}

Keywords: Andes; altitudinal range; climate change; species distribution; tree diversity; tropical montane forest.

\section{Introducción}

El Perú está considerado como uno de los países tropicales megadiversos del mundo, y dentro de su complejidad ecológica y topográfica destaca la cordillera de los Andes, que está considerada como una de las zonas más diversas dentro de las ecoregiones críticas o los puntos calientes (hotspot) de biodiversidad en los trópicos (Myers et al. 2000). Las exploraciones botánicas en el Perú empezaron en el siglo XVIII con la de Hipólito Ruiz López y José Pavón que después de épicas campañas regresaron a España con más de 3000 especímenes de plantas (Alvarez Lopez 1953). Alexander von Humboldt y Aimé Bonpland durante el siglo diecinueve realizaron valiosos aportes a la flora peruana, dejando los fundamentos para la ecología vegetal y la biogeografía moderna (Egerton 1970, Lomolino 2001). La Flora de Perú escrita por Macbride (1936-1970) fue la revisión botánica más extensa y comprehensiva para el Perú hasta finales de los años 90s, siendo el documento con más tratamientos botánicos para la época. En la década de los años 90, Brako y Zaruchi publicaron el Catalogo de las Angiospermas y Gimnospermas del Perú (Brako \& Zarucchi 1993), una lista anotada valiosísima para un mejor conocimiento de la flora nacional y además para un mejor conocimiento del endemismo, la distribución de especies y la ecología vegetal. Uno de los últimos aportes y más completos a nuestra flora lo constituye la Guía Ilustrada de Arboles de Perú, donde se estima que para el Perú existen aproximadamente 6350 especies de árboles, cifra que puede incrementarse al menos en un $10 \%$ con el mejoramiento del conocimiento botánico (Pennington et al. 2004).

Los Andes peruanos empezaron a tener mayores incursiones botánicas en el siglo diecinueve, con exploradores, naturalistas y científicos como Hugh Weddell, Augusto Weberbauer y Antonio Raimondi. En el siglo veinte surge la botánica peruana siendo sus mejores representantes Fortunato L. Herrera y Cesar Vargas y Ramón Ferreyra (León et al. 2006). En las últimas décadas se produjeron múltiples listados y adiciones a la flora de los Andes, incrementando nuestro conocimiento en los diferentes grupos de Gimnospermas y Angiospermas (Galiano 1993, Vargas 1994, Ulloa Ulloa et al. 2004, Tupayachi H. 2005, León et al. 2006, Rodríguez et al. 2006, Monteagudo Mendoza \& Huamán Gue- rrero 2010). A estas se suman las campañas botánicas realizadas por el Jardín Botánico de Missouri y The Field Museum, en especial con la publicación de las Las Guias de Plantas Tropicales (http://fm2.fieldmuseum.org/plantguides/), una notable contribución liderada por Robin Foster. Finalmente, es invaluable la contribución de A. Gentry (1996) a la flora de los Andes con sus múltiples excursiones y publicaciones en particular el texto A Field Guide to the Families and Genera of Woody Plants of North West South America: (Colombia, Ecuador, Peru).

Sin embargo, la poca accesibilidad a estos complejos paisajes naturales, el alto endemismo, la alta diversidad y los estrechos rangos altitudinales de las especies vegetales hacen que estos ecosistemas montańosos sean subestimados en riqueza y diversidad de especies vegetales, uno de ellos es el Parque Nacional del Manu.

Investigadores, naturalistas y exploradores han venido acumulando una extensiva información de la flora en el Parque Nacional del Manu desde los años 70, pero muchas de estas se concentran principalmente en los bosques bajos, específicamente en las zonas de Cocha Cashu y Pakitza (Cano et al. 1995, Wilson \& Sandoval 1996) un ejemplo de esta información es la plataforma virtual de Manu plants (http://manuplants.org/) donde se encuentran sistematizadas 970 especies de árboles y arbustos de selva baja. Recientemente se viene incrementando el conocimiento botánico en los bosques montanos del Parque Nacional del Manu, pero en forma dispersa y limitada como las múltiples guías ilustradas realizadas por R. Foster (http://fm2. fieldmuseum.org/plantguides/) y el estudio de vegetación realizado en la parte alta del bosque montano del Parque Nacional del Manu (Cano et al. 1995). Recientemente, Atrium (http:// atrium.andesamazon.org/) viene incrementando el conocimiento botánico en el Parque con su plataforma de biodiversidad de libre acceso.

La información científica en los bosques montanos sigue siendo escasa, es así que se presenta esta lista anotada de árboles y grupos afines de los bosques montanos de la Parque Nacional del Manu, en base a inventarios en parcelas permanentes y múltiples exploraciones en diferentes puntos de la zona con el 


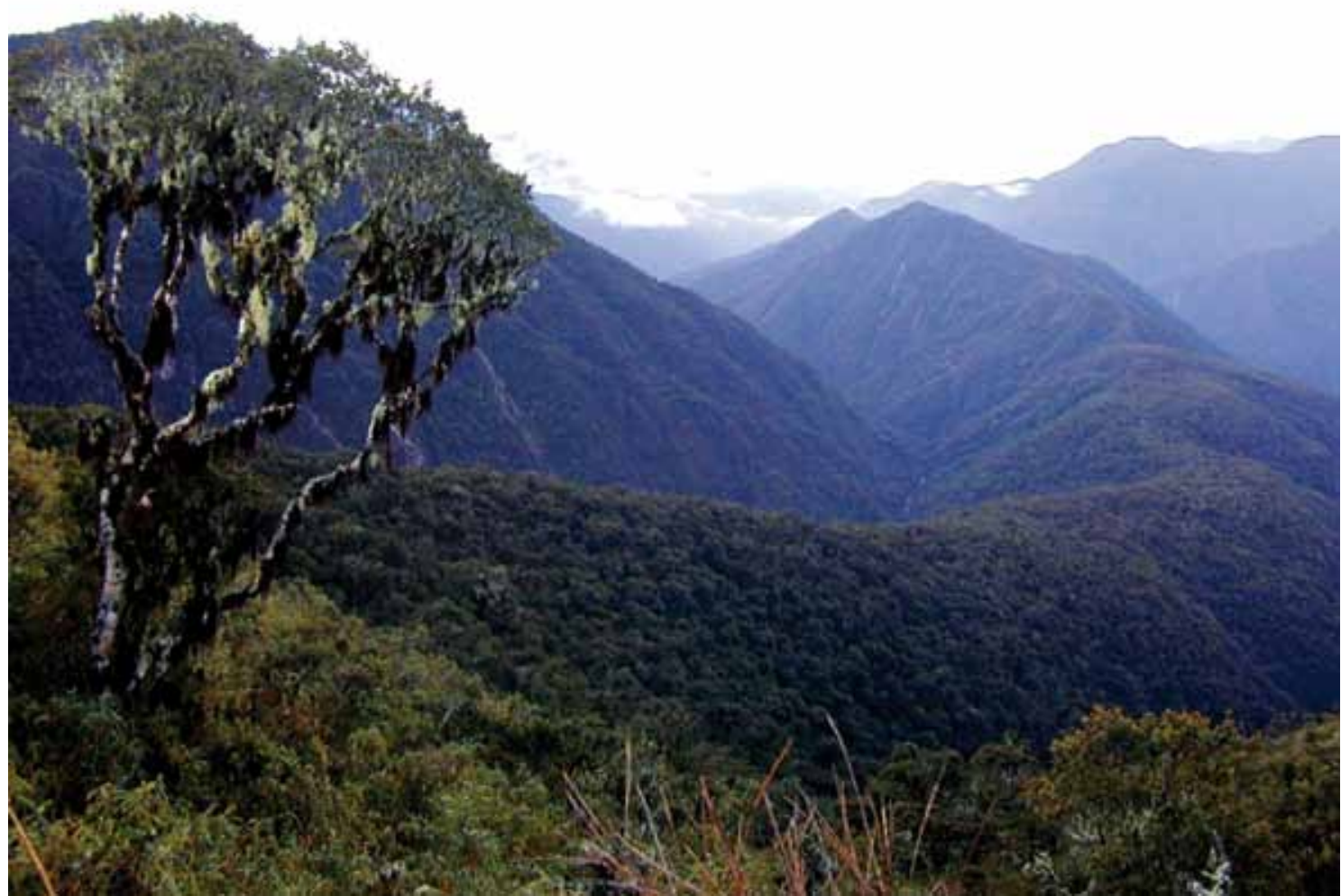

Figura 1. Vista panorámica del transecto altitudinal de Trocha Unión en el Valle de Kosñipata, ocho parcelas permanentes se encuentran instaladas en la cresta de esta montaña que va desde los $1800 \mathrm{~m}$ hasta los 3600 m. Credito de foto: W. Farfan Rios.

fin de incrementar el conocimiento empírico y tener un mejor entendimiento de la flora arbórea y afines en estos ecosistemas neotropicales megadiversos.

\section{Métodos}

El área de estudio se encuentra el Parque Nacional del Manu (PNM) y su zona de amortiguamiento, ubicado en la región andina al sureste peruano. El PNM es una de las área protegidas del Perú con mayor extensión geográfica y en 1987 fue declarada como Patrimonio Natural de la Humanidad por la UNESCO (SERNANP 2013). La excepcional biodiversidad de esta área natural protegida se expande desde los bosques lluviosos de tierras bajas a $-200 \mathrm{~m}$ de elevación pasando por los bosques montanos (Fig. 1) hasta legar a los pajonales húmedos alto-andinos a más de $-4000 \mathrm{~m}$ de elevación. El clima en el parque viene siendo estudiado a una escala local, ahora se sabe que existe una estacionalidad marcada con la presencia de niebla constante en todo el ańo. La precipitación anual presenta una alta variabilidad, se estima entre $>5000 \mathrm{~mm}$ anuales a elevaciones bajas a $890 \mathrm{~m}$ hasta $<1000 \mathrm{~mm}$ anuales a los $4130 \mathrm{~m}$ de altitud, la temperatura decrece linealmente a lo largo de la gradiente altitudinal entre los bosques lluviosos bajos y los pajonales húmedos altos, en un rango de 24 a $7.7{ }^{\circ} \mathrm{C}$ respectivamente (Rapp 2010, Rapp \& Silman 2012).

El presente listado incluye a todos los individuos de porte arbóreo $\geq 10 \mathrm{~cm}$ de diámetro a $1.3 \mathrm{~m}$ sobre el suelo, con un tallo principal y que se encuentren libremente erguidos, se incluyen especies nativas presentes en los bosques montanos no perturbados. Dentro de los grupos funcionales muestreados se tienen a los árboles, helechos arbóreos, palmeras y lianas. Esta lista es esta basada en la información procedente de la red de parcelas permanentes de una hectárea establecidas por el grupo de investigación Andes Biodiversity and Ecosystem Research Group - ABERG (http://www.andesconservation.org/) a lo largo de un gradiente de elevación en el Parque Nacional del Manu, para efectos de esta publicación se incluyen todos los registros desde los bosques sub-montanos a $800 \mathrm{~m}$ de elevación hasta la línea de bosque a $3625 \mathrm{~m}$ en base a 21 parcelas permanentes y exploraciones en áreas adyacentes desde el año 2003.

El material estudiado corresponde a las colecciones realizadas en las parcelas permanentes y áreas adyacentes, las cuales están depositadas en herbarios peruanos (CUZ, HUT, MOL, USM) con duplicados en herbarios extranjeros (DAV, MO, F, WFU). El proceso de identificación de este material botánico continúa con la colaboración de diferentes taxónomos pertenecientes a varias instituciones. En este catálogo se tienen a las especies reconocidas por la APG III (Angiosperm Phylogeny Group. 2009), se usó la herramienta TNRS versión 3.2 (http://tnrs.iplantcollaborative. org) para la corrección semántica, posibles errores en la escritura y sobre todo para la corrección de los nombres falsos (Boyle et al. 2013). Para la distribución geográfica, los rangos altitudinales se usaron los sitios web de Tropicos (http://www.tropicos.org), "Plant List" (http://www.theplantlist.org), JSTOR - Global Plants (http://plants.jstor.org/), la plataforma de biodiversidad "Atrium" (http://atrium.andesamazon.org/) y la plataforma "Global Biodiversity Information Facility - GBIF" (http:// 
www.gbif.org/(León et al. 2006) y la lista $A$ Regional Red List of Montane Tree Species of the Tropical Andes (Tejedor Garavito et al. 2014) para catalogar a las especies endémicas.

\section{Resultados y discusiones}

De las múltiples exploraciones y en base a las parcelas permanentes en el PNM se tienen resultados preliminares traducidos en 95 familias, 272 géneros y 1108 especies de árboles y afines, un número alto de especies considerando una distancia geográfica corta de $-20 \mathrm{~km}$ a lo largo de la gradiente altitudinal. El $60.3 \%$ (668 especies) del total de especies se encuentran identificadas al nivel de especie, el 43\% (287 especies) de las especies determinadas son registros nuevos para la región Cusco, 15 especies son registros nuevos para la flora Peruana, 40 especies son endémicas para Perú y 30 potenciales especies nuevas para la ciencia. En adición, se amplió el rango altitudinal para 302 especies (45.2\%) del total de especies determinadas. Finalmente, se registraron 12 especies arbóreas dentro la categoría de conservación "globalmente amenazadas" según la lista roja de especies arbóreas montanas en los Andes Tropicales (Tejedor Garavito et al. 2014) las que corresponden a: Alchornea anamariae Secco, Axinaea glandulosa Ruiz \& Pav. ex D. Don, Ilex sessiliflora Triana \& Planch., Brunellia brunnea J.F. Macbr., Brunellia inermis Ruiz \& Pav., Freziera dudleyi A.H. Gentry, Oreopanax ruizii Decne. \& Planch. ex Harms, Persea brevipes Meisn., Prunus pleiantha Pilg., Schefflera inambarica Harms, Sessea dependens Ruiz \& Pav., Symplocos reflexa A. DC. La mayor parte de los registros arbóreos en el área de estudio se encontraron en la zona premontana a montana, aproximadamente entre los $1000-2500$ m de elevación.

Existe una única lista de plantas con flores para los bosques montanos en el Parque Nacional del Manu que incluye hierbas, arbustos y árboles la cual está limitada a familias y géneros (Cano et al. 1995) y que convierte a este estudio en un aporte importante al conocimiento de la flora del Parque Nacional del Manu y de los bosques montanos neotropicales en general. En la Tabla 1, la información florística se encuentra sistematizada alfabéticamente, en el orden de Pteridophyta, Gymnospermae y Angiospermae, anotada por rangos de elevación registrados en la zona de estudio, la distribución geográfica en Perú y endemismo, los registros nuevos para la región Cusco están indicados con $\left(^{*}\right)$, los registros nuevos para la flora Peruana están indicados con $\left({ }^{* *}\right)$, y la ampliación de los rangos de distribución indicados con $(\wedge)$ además, se indican las potenciales especies nuevas como $(\&)$ las cuales vienen siendo revisadas y corresponden a: Annonaceae [Guatteria (1), Rollinia (1), P. Mass], Araliaceae [Dendropanax (1), Oreopanax (1), Schefflera (1), J. Wen com pers.), Ebenaceae [Lissocarpa (1), R. Liesner], Euphorbiaceae [Alchornea (1), N. Hensold; Micrandra (1) K. Wurdack], Lacistemataceae [Lozania (1), R. Liesner], Lauraceae [Endlicheria (1), Nectandra (3), Ocotea (3), Persea (2), H. van der Werff com pers.], Melastomataceae [Miconia (2), F. Michelangeli com pers.] Myrtaceae [Myrcia (1) L. Kawasaki], Moraceae [Ficus (1)], Rubiaceae [Psychotria (2) C.M. Taylor com pers.], Sabiaceae [Meliosma (1), R. Liesner], Sapotaceae [Chrysophyllum (1), Sarcaulus (1), R. Foster], Symplocaceae [Symplocos (1)], Urticaceae [Cecropia (1)], Vochysiaceae [Vochysia (1), L. Kawasaki].

Del total de especies encontradas en el área de estudio, el $39.7 \%$ se encuentra como morfoespecies debido principalmente a que las colecciones se encuentran en estado estéril haciendo difícil su identificación ya sea por comparación en los herbarios o usando claves dicotómicas, estas más de 400 morfoespecies tienen el potencial de pertenecer a otros taxa y probablemente puedan ser especies nuevas para la ciencia. Lauraceae, Fabaceae, Melastomataceae y Cyatheaceae fueron las familias que presentaron el mayor número de morfoespecies y son las familias que ameritan mayor atención en su taxonomía. Estos resultados es una muestra de lo poco estudiados que se encuentran estos ecosistemas montañosos, en este caso en términos de especies vegetales de porte arbóreo, esta escases de material botánico en los herbarios hace difícil la determinación de especies, como ejemplo tenemos a Guatteria terminalis R.E. Fr. (Anexos 1) colectada por primera vez en Puno por Lechler en Agosto de 1854, y después de 149 años fue colectada en el Parque Nacional del Manu entre los 1800-2250 m de elevación, cuya determinación se realizó después de 5 años. Así mismo Clusiacae, Primulaceae y Sabiaceae son las familias poco estudiadas en este tipo de bosques por las colecciones limitadas y la falta de especialistas.

En Anexos, se presentan laminas ilustradas con ejemplos de especies endémicas [Guatteria terminalis (Anexo 1), Cyathea multisegmenta y Ocotea glabriflora (Anexo 2) y Symplocos psiloclada (Anexo 3)]; potencial especie nueva [Schefflera sp.1_158WHH (Anexo 1)]; especies comunes [Prunus integrifolia, Symplocos psiloclada, (Anexo 3)] y Retrophyllum rospigliosii (Anexo 3) como una especie rara para la zona de estudio.

Los Bosques montanos del Parque Nacional del Manu son un repositorio natural que engloba una alta biodiversidad, estos resultados, principalmente las potenciales nuevas especies, los nuevos registros y la ampliación de rangos altitudinales convierten este laboratorio natural en una de las áreas naturales protegidas más importantes de nuestro país; y generara un entendimiento comprensivo sobre la distribución de especies y además, ayuda a responder preguntas fundamentales en la biogeografía y la ecología vegetal moderna contribuyendo así a las políticas de conservación y protección de estos ecosistemas Andinos Tropicales.

\section{¿Es importante seguir colectando material botánico?}

Más colecciones botánicas son necesarias. En los trópicos nueve de cada diez especies se encuentran pobremente colectadas con $<20$ registros (Feeley and Silman 2011), la escases de esta información básica pero fundamental tiene mayores implicaciones en el mejor entendimiento de los patrones de distribución de especies y su conservación, así mismo, estos registros mínimos hace que las especies sean casi invisibles para el modelamiento moderno de distribución de especies limitando las simulaciones en los diferentes escenarios del calentamiento global. Más esfuerzos son necesarios para incrementar las colecciones geo-referenciadas de especies vegetales, estas combinadas con la digitalización de las colecciones existentes en los herbarios y museos se podrá predecir el impacto del cambio climático en las especies tropicales (Feeley et al. 2011, Feeley \& Silman 2011).

La revolución tecnológica en la ecología moderna, como es el caso del sensoramiento remoto combinado con datos de espectrometría a nivel foliar ahora permiten realizar muestreos (para la identificación de especies arbóreas) de conjunto de especies en múltiples escalas geográficas, desde el nivel de individuo hasta 
la escala de paisaje (Asner and Martin 2009, Silman 2014), sin embargo, estas técnicas modernas aún están limitadas por la presencia de morfoespecies en los bosques tropicales.

Actualmente, gracias a los múltiples inventarios y colecciones botánicas se estima que existen aproximadamente 16000 especies de árboles en la Amazonia de los cuales se tienen nombres para al menos 10,000 especies, y solamente 5,000 de ellas fueron encontradas en redes de parcelas permanentes (ter Steege et al. 2013). ¿Cuantas especies arbóreas existen aproximadamente en los Andes? Es una pregunta trascedental, debido a la escasa y dispersa información botánica y ecológica que existe de estos majestuosos ecosistemas montañosos.

\section{Agradecimientos}

Este estudio fue posible gracias al apoyo de Andes Biodiversity and Ecosystem Research Group (ABERG), los autores expresan su agradecimiento a los directores y curadores de los herbarios: Herbario Vargas (CUZ), Herbario del Museo de Historia Natural- Universidad Nacional Mayor de San Marcos (USM), Herbario Nacional de Bolivia (LPB), al Field Museum (F) y al Herbario del Missouri Botanical Garden (MO) por el acceso a las colecciones botánicas. Agradecemos a GBIF, JSTOR, TROPICOS, TNRS y a todas las instituciones que hicieron posible que los datos botánicos estén disponibles para su publicación. Agradecimientos especiales a todos los estudiantes de la Universidad Nacional de San Antonio Abad del Cusco por el extraordinario esfuerzo en las diferentes campañas de campo, en especial a Marlene Mamani, Darcy Galiano, Vicky Huamán, Juan A. Jibaja, Israel Cuba, Alex Nina, W. Huaraca, Erickson Urquiaga, Tatiana Boza, Jhoel Delgado, A. Rozas, B.G. Valencia, Carlos A. Salas, Rudy S. Cruz, Percy Chambi y Luis Imunda. A la Dirección General de Gestión Sostenible del Patrimonio Forestal y de Fauna Silvestre (DGFFS), al Servicio de Áreas Naturales Protegidas por el Estado (SERNANP) y a la Jefatura del Parque Nacional del Manu por las respectivas autorizaciones de investigación y el apoyo logístico. A Pantiacolla tours, a la Asociación para la Conservación de la Cuenca Amazónica y al Albergue Gallito de las Rocas por proveer apoyo logístico. Así mismo, nuestro reconocimiento y agradecimiento a Robin Foster, Paul Mass, Ronald Liesner, Jun Wen, Kenneth Wurdack, Nancy Hensold, Henk van der Werff, Fabian A. Michelangeli, Lucia Kawasaki y Charlote M. Taylor por el apoyo en las determinaciones botánicas.

\section{Literatura citada}

Alvarez Lopez E. 1953. Algunos aspectos de la obra de Ruiz y Pavon. Anales Inst. Bot. Cavanilles 12:5-113.

Angiosperm Phylogeny Group. 2009. An update of the Angiosperm Phylogeny Group classification for the orders and families of flowering plants: APG III. Botanical Journal of the Linnean Society 161:105-121.

Asner G.P. \& R.E. Martin. 2009. Airborne spectranomics: mapping canopy chemical and taxonomic diversity in tropical forests. Frontiers in Ecology and the Environment 7:269-276.

Boyle B., N. Hopkins, Z. Lu, J. A. Raygoza Garay, D. Mozzherin, T. Rees, et al. 2013. The taxonomic name resolution service: an online tool for automated standardization of plant names. BMC bioinformatics 14:16.

Brako L., \& J. Zarucchi. 1993. Catálogo de las Angiospermas y Gimnospermas del Perú. Monographs in Systematic Botany from the Missouri Botanical Garden 45: 763-867

Cano A., K.R. Young, B. Leon, \& R.B. Foster. 1995. Composition and diversity of flowering plants in the upper montane forest of Manu National Park, Southern Peru. Pages 271-280 In:
Churchill S.P., H. Balslev, E. Forero, \& J.L. Luteyn, editors. Biodiversity and Conservation of Neotropical Montane Forests: Proceedings of the Neotropical Montane Forest. New York Botanical Garden Pr Dept, United states of America.

Egerton F.N. 1970. Humboldt, Darwin, and Population. Journal of the History of Biology 3:325-360.

Feeley K.J. \& M.R. Silman. 2011. The data void in modeling current and future distributions of tropical species. Global Change Biology 17:626-630. http://dx.doi.org/10.1111/j.13652486.2010.02239.x

Feeley K.J., M.R. Silman, M.B. Bush, W. Farfan, K.G. Cabrera, et al. 2011. Upslope migration of Andean trees. Journal of Biogeography 38:783-791. http://dx.doi.org/10.1111/j.13652699.2010.02444.x

Galiano S.W. 1993. Diversidad Biológica en los Andes Sur-Orientales. Rev. Q'ente.CC. BB. Cusco.

Gentry A.H. 1996. A Field Guide to the Families and Genera of Woody Plants of North West South America: (Colombia, Ecuador, Peru). University Of Chicago Press. 920pp

León B., J. Roque, C. Ulloa Ulloa, N. Pitman, P. M. Jørgensen, and A. Cano. 2006. El libro rojo de las plantas endémicas del Perú. Revista Peruana de Biología 13:1-965.

Lomolino M.V. 2001. Elevation gradients of species-density: historical and prospective views. Global Ecology and Biogeography 10:3-13. http://dx.doi.org/10.1046/j.1466$822 x .2001 .00229 . x$

Monteagudo Mendoza A.L. \& M. Huamán Guerrero. 2010. Catálogo de los arboles y afines de la Selva Central del Perú. Arnaldoa 17:203-242.

Myers N., R. A. Mittermeier, C. G. Mittermeier, G. A. B. da Fonseca, and J. Kent. 2000. Biodiversity hotspots for conservation priorities. Nature 403:5. http://dx.doi. org/10.1038/35002501

Pennington T., C. Reynel \& A. Daza. 2004. Illustrated guide to the trees of Peru. D. Hunt Publisher, England. 848 pp.

Rapp J. 2010. Climate control on plant performance across an Andean altitudinal gradient. Dissertation Degree of Doctor of Philosophy. Wake Forest University, Winston Salem, North Carolina, USA. http://hdl.handle.net/10339/30425

Rapp J.M. \& M.R. Silman. 2012. Diurnal, seasonal, and altitudinal trends in microclimate across a tropical montane cloud forest. Climate Research 55:17-32. http://dx.doi. org/10.3354/cr01127

Rodríguez E.F., R. Vásquez, R. Rojas, G. Calatayud, B. Leon, \& J. Campos. 2006. Nuevas adiciones de angiospermas a la flora del Perú. Revista Peruana de Biología 13:129-138. http:// dx.doi.org/10.15381/rpb.v13i1.1776

SERNANP (Servicio Nacional de Áreas Naturales Protegidas por el Estado). 2013. Parque Nacional del Manu, Plan Maestro, Diagnostico, 2013-2018. Lima. Servicio Nacional de Areas Naturales Protegidas por el Estado - SERNANP. 138 pp

Silman M.R. 2014. Functional megadiversity. Proceedings of the National Academy of Sciences of the United States of America 111:5763-4. http://dx.doi.org/10.1073/pnas.1402618111

Ter Steege H., N.C.A. Pitman, D. Sabatier, C. Baraloto, R. P. Salomão, et al. 2013. Hyperdominance in the Amazonian tree flora. Science (New York, N.Y.) 342:1243092. http://dx.doi. org/10.1126/science.1243092

Tejedor Garavito N., E. Álvarez Dávila, S. Arango Caro, A. Araujo Murakami, A. Baldeón, et al. 2014. A Regional Red List of Montane Tree Species of the Tropical Andes: Trees at the top of the world. Richmond. Botanic Gardens Conservation International. Pp. 56.

Tupayachi H., A. 2005. Flora de la Cordillera de Vilcanota. Arnaldoa 12:126-144.

Ulloa Ulloa, C., J. L. Y. Zarucchi, and B. León. 2004. Diez años de adiciones a la flora del Perú: 1993-2003. Arnaldoa Edición Especial Nov.2004:1-142.

Vargas, C. 1994. Flora del Sur del Peru: Catálogo Sistemático del Herbario Vargas (CUZ). FCB - UNSAAC. GTZ:393 pp.

Wilson D.E. \& A. Sandoval (Eds). 1996. Manu: the biodiversity of south-eastern Peru. Washington D.C. Smithsonian Institution. $674 \mathrm{pp}$. 
Tabla 1. Lista de especies arbóreas y afines en los bosques montanos del sureste peruano.

\section{PTERIDOPHYTA}

CYATHEACEAE Kaulf.

1. Alsophila cuspidata (Kunze) D.S. Conant $1250-2250 \mathrm{~m}$. ^ K. Garcia, et al. 741 (CUZ, MOL) AM, CU, HU, LO, MD, PA, PU, UC

2. Alsophila erinacea (H. Karst.) D.S. Conant $1250-2500 \mathrm{~m}$

K. Garcia, et al. 211 (CUZ, MO, USM, WFU) CA, CU, MD, PA, SM

3. Alsophila sp. 1 (743KGC)

$1250 \mathrm{~m}$.

K. Garcia, et al. 743 (CUZ, USM)

4. Alsophila sp. 2 (953KGC) $1750 \mathrm{~m}$.

K. Garcia, et al. 953 (USM)

5. Cyathea andina (H. Karst.) Domin $850 \mathrm{~m} .{ }^{*}$

W. Farfan, et al. 5563 (CUZ) JU, LO, MD, PA

6. Cyathea caracasana (Klotzsch) Domin $2000-3250 \mathrm{~m}$.

K. Garcia, et al. 140 (CUZ, MO) AM, CA, CU, JU, PA, SM

7. Cyathea delgadii Sternb. $2250-3250 \mathrm{~m}$.

K. Garcia, et al. 147 (CUZ, HUT, MO, USM, WFU) AM, CA, CU, HU, JU, MD, PA, SM

8. Cyathea divergens Kunze $2500-3450 \mathrm{~m} . \wedge$ K. Garcia, et al. 146 (CUZ, MO) CU, JU, PA

9. Cyathea lechleri Mett. $1800-2750 \mathrm{~m}$. K. Garcia, et al. 153 (CUZ, MO) AM, CA, CU, PA, PU, SM

10. Cyathea multisegmenta R.M. Tryon 1800 - $2250 \mathrm{~m}$. Endémico K. Garcia, et al. 208 (CUZ, HUT, MO, USM, WFU) CU, PA

11. Cyathea pallescens (Sodiro) Domin $2890-3450$ m. *^ K. Garcia, et al. 145 (CUZ, MO, USM, WFU) AM, JU, PA, SM, UC

12. Cyathea ruiziana Klotzsch $2250 \mathrm{~m}$. Endémico K. Garcia, et al. 625 (CUZ) CU, JU, PA, SM

13. Cyathea sp. 1 (238KGC) $2250-3000 \mathrm{~m}$

K. Garcia, et al. 238 (CUZ, MO)

14. Cyathea sp. 10 (1568KGC) $2500 \mathrm{~m}$. K. Garcia, et al. 1568 (USM)

15. Cyathea sp. 11 (1567KGC) $2500 \mathrm{~m}$.

K. Garcia, et al. 1567 (USM)

16. Cyathea sp. 12 (1597KGC) $2250 \mathrm{~m}$.

K. Garcia, et al. 1597 (USM)

17. Cyathea sp. 13 (726KGC)

$1250-1500 \mathrm{~m}$.

K. Garcia, et al. 726 (USM)

18. Cyathea sp.14 (571KGC) $1500 \mathrm{~m}$.

K. Garcia, et al. 571 (USM)

19. Cyathea sp. 15 (1043KGC) $1500-1750 \mathrm{~m}$

K. Garcia, et al. 1043 (USM)

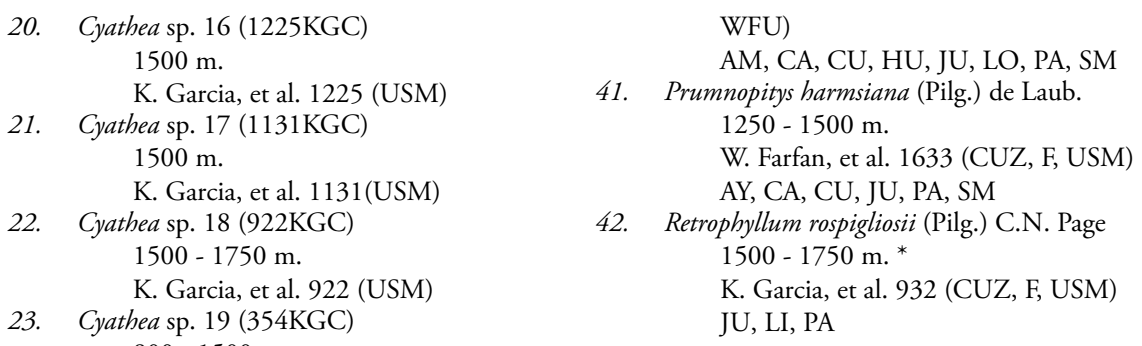

WFU)

AM, CA, CU, HU, JU, LO, PA, SM

41. Prumnopitys harmsiana (Pilg.) de Laub. $1250-1500 \mathrm{~m}$.

W. Farfan, et al. 1633 (CUZ, F, USM) AY, CA, CU, JU, PA, SM

42. Retrophyllum rospigliosii (Pilg.) C.N. Page $1500-1750$ m. *

K. Garcia, et al. 932 (CUZ, F, USM)

$$
\text { JU, LI, PA }
$$

\section{GYMNOSPERMAE}

\section{PODOCARPACEAE Endl.}

40. Podocarpus oleifolius D. Don ex Lamb. $1800-3000 \mathrm{~m}$.

W. Farfan, et al. 3322 (CUZ, USM,

\section{ANGIOSPERMAE}

ACTINIDIACEAE Gilg \& Werderm.

43. Saurauia glabra (Ruiz \& Pav.) Soejarto

$1750 \mathrm{~m} . * \wedge$

K. Garcia, et al. 1051 (USM, WFU)

CA, PA

44. Saurauia peruviana Buscal.

1500 - 1750 m. *

K. Garcia, et al. 1084 (USM, WFU)

AM, CA, LL, PA, SM

45. Saurauia sp. 3 (2547WFR) $1750 \mathrm{~m}$.

W. Farfan, et al. 2547 (CUZ, F, USM, WFU)

ADOXACEAE E. Mey.

46. Viburnum ayavacense Kunth $1500-2250 \mathrm{~m} . * \wedge$

W. Farfan, et al. 1016 (CUZ, F, MO, USM)

CA, JU, LA, PI

47. Viburnum hallii (Oerst.) Killip \& A.C. Sm. $1500-2250 \mathrm{~m}$.

K. Garcia, et al. 1030 (CUZ, F, USM) $\mathrm{AM}, \mathrm{CA}, \mathrm{CU}, \mathrm{HU}, \mathrm{JU}, \mathrm{PA}$

48. Viburnum reticulatum (Ruiz \& Pav. ex Oerst.) Killip

$2890-3000 \mathrm{~m} \cdot * \wedge$

W. Farfan, et al. 4683 (MOL, WFU) AM, CA, SM

\section{ALZATEACEAE S.A. Graham}

49. Alzatea verticillata Ruiz \& Pav. $1750-2250 \mathrm{~m}$. ^ W. Farfan, et al. 1102 (CUZ, MO, USM)

CA, CU, JU, PA, UC

ANACARDIACEAE R. Br $800-1000 \mathrm{~m}$.

W. Farfan, et al. 4128 (MOL, WFU) AM, CU, HU, JU, LO, MD, PA, SM, $\mathrm{UC}$

51. Tapirira obtusa (Benth.) J.D. Mitch.

$1500-1750 \mathrm{~m} . * \wedge$

K. Garcia, et al. 1119 (CUZ, F, MO, USM) AM, LO, MD, PA, SM

52. Tapirira sp. 1 (4573WFR) $1250-1800 \mathrm{~m}$. W. Farfan, et al. 4573 (MOL, WFU)

\section{ANNONACEAE Juss.}

53. Annona excellens R.E. Fr.

$850 \mathrm{~m} . * \wedge$

W. Farfan, et al. 5467 (CUZ) $\mathrm{LO}, \mathrm{MD}, \mathrm{SM}$

54. Annona papilionella (Diels) H. Rainer $1250-1500 \mathrm{~m} . \wedge$

K. Garcia, et al. 628 (CUZ, USM, F, WFU)

AM, CU, HU, LO, MD, PA, SM, UC

55. Annona williamsii (Rusby ex R.E. Fr.) H. Rainer $1250-1500 \mathrm{~m} . \wedge$

W. Farfan, et al. 3977 (CUZ, F, USM, WFU)

$\mathrm{CU}, \mathrm{SM}, \mathrm{MD}$
50. Tapirira guianensis Aubl. 
Tabla 1. Lista de especies arbóreas y afines en los bosques montanos del sureste peruano.

\begin{tabular}{|c|c|c|c|c|c|}
\hline \multirow[t]{3}{*}{56.} & $\begin{array}{l}\text { Guatteria dielsiana R.E. Fr. } \\
\quad 850 \mathrm{~m} . \wedge\end{array}$ & & $\begin{array}{l}\text { WFU) } \\
\text { AM, CU, LO, MD, PA, SM, UC }\end{array}$ & & $\begin{array}{l}\text { J. E. Silva, et al. } 642 \text { (CUZ, MO, } \\
\text { USM) }\end{array}$ \\
\hline & W. Farfan, et al. 5463 (CUZ) & 74. & Rollinia sp. 1 (1138WFR) & 91. & Ilex gabrielleana Loizeau \& Spichiger \\
\hline & CU, LO, MD, SM, UC & & $2250 \mathrm{~m} . \&$ & & $1500-1750$ m. *^ \\
\hline \multirow[t]{4}{*}{57.} & $\begin{array}{l}\text { Guatteria duodecima Maas \& Westra } \\
\qquad 1250 \text { - } 1750 \mathrm{~m} . * \wedge\end{array}$ & & $\begin{array}{l}\text { W. Farfan, et al. } 1138 \text { (CUZ, MO, F, } \\
\text { USM) }\end{array}$ & & $\begin{array}{l}\text { K. Garcia, et al. } 939 \text { (USM, MO) } \\
\text { LO, AM, SM, PA }\end{array}$ \\
\hline & W. Farfan, et al. 1585 (CUZ, F, USM, & 75. & sp. 1 (1934WFR) sp. 1 (1934WFR) & 92. & Ilex guayusa Loes. \\
\hline & WFU) & & $1250 \mathrm{~m}$ & & $2500 \mathrm{~m} .{ }^{* \wedge}$ \\
\hline & LO, MD, PA, SM & & W. Farfan, et al. 1934 (CUZ, MO, F, & & W. Farfan, et al. 947 (CUZ, MO, \\
\hline \multirow[t]{4}{*}{58.} & $\begin{array}{l}\text { Guatteria glauca Ruiz \& Pav. } \\
\qquad 1500-1800 \mathrm{~m} .\end{array}$ & 76. & $\begin{array}{l}\text { USM) } \\
\text { Unonopsis guatterioides R.E. Fr. }\end{array}$ & & $\begin{array}{l}\text { USM, WFU) } \\
\text { AM, CA, LA, L( }\end{array}$ \\
\hline & W. Farfan, et al. 1071 (CUZ, F, USM, & & $1250-1500 \mathrm{~m}^{* \wedge}$ & 93. & Ilex karstenii Loes. \\
\hline & WFU) & & , et al. 593 (CUZ, MO, F, & & $3450 \mathrm{~m} .{ }^{* \wedge}$ \\
\hline & AM, CA, CU, HU, LO, MD, PA, SM & & USM) & & W. Farfan, et al. 2967 (USM, WFU) \\
\hline \multirow[t]{5}{*}{59.} & Guatteria guentheri Diels & & AM, HU, LO, MD, SM, UC & & CA \\
\hline & $1250^{\circ}$ & 77. & Unonopsis spectabilis Diels & 94. & Ilex laurina Kunth \\
\hline & K. Garcia, et al. 793 (CUZ, F, USM, & & & & $0 \mathrm{~m} .{ }^{*}$ \\
\hline & WFU) & & an, et al. 5454 (CUZ) & & t al. 3086 (CUZ, F, USM, \\
\hline & CU, LO, MD, PA, PU & & A, PU, SM, UC & & WFU) \\
\hline \multirow[t]{4}{*}{60.} & Guatteria & 78. & Xylopia benthamii R.E. Fr. & & AM, CA, MD, PA, SM \\
\hline & & & & 95. & Ilex microd \\
\hline & t al. 1536 (CUZ, F, USM, & & an, et al. 1502 (CUZ, MO) & & \\
\hline & WFU) & & Cl & & 99 (CUZ, MO, \\
\hline \multirow[t]{3}{*}{61.} & Guatteria sp. 12 (1129KGC) & 79. & Xylopia cal & & \\
\hline & 1500 & & * & 96. & Ilex nayan \\
\hline & a, et al. 1129 (USM, WFU) & & fan, et al. 1298 (CUZ, MO, F, & & \\
\hline \multirow[t]{3}{*}{62.} & Guatteria sp. 14 (3151WFR) & & USM) & & W. Farfan, et al. 1325 (CUZ, F, USM, \\
\hline & & & MD, PA & & \\
\hline & 3151 (MOL, WFU) & APO & CYNAC & & LO \\
\hline \multirow[t]{3}{*}{63.} & Guatteric & 80. & celsum Benth. & 97. & Ilex ner \\
\hline & & & & & \\
\hline & n, et al. 3257 (MOL, WFU) & & in, et al. 5496 (USM) & & al. 1093 (CUZ, F, USM, \\
\hline \multirow[t]{3}{*}{64.} & Guatteria: & & & & \\
\hline & & 81. & Aspidos & & \\
\hline & in, et al. 1180 (CUZ, MO) & & & 98. & Xlanch. \\
\hline \multirow[t]{3}{*}{65.} & Guatteria sp. 3 (3 & & an, et al. 5488 (CUZ) & & 3000 \\
\hline & & & & & 836 (CUZ, DAV, \\
\hline & 3152 (MOL, WFU) & 82. & Aspidosp & & \\
\hline \multirow[t]{4}{*}{66.} & Guatteria s & & $0 \mathrm{~m} . \wedge$ & & $\mathrm{Cl}$ \\
\hline & & & Irfan, et al. 1397 (CUZ, MO, & 99. & Ilex sp. 1 \\
\hline & 216 (CUZ, F, USM, & & WFU) & & \\
\hline & & & $\mathrm{O}, \mathrm{MD}, \mathrm{UC}$ & & (CUZ \\
\hline 67. & rminalis R.E. Fr. & 83. & Aspidost & & \\
\hline & & & & 100. & Ilex sp. 8 \\
\hline & an, et al. 1112 (CUZ, DAV, & & t al. 698 (CUZ, F, USM) & & \\
\hline & & 84. & Aspidosp & & $\mathrm{M}$, \\
\hline & PU & & & & \\
\hline 68. & Guatteria tomentosa Rusby & & 078 (CUZ, F) & 101. & oclada Loes. \\
\hline & & 85. & a sp. 4 (5478WFR) & & *** \\
\hline & fan, et al. 2260 (CUZ, F, USM, & & & & an, et al. 853 (CUZ, HUT, \\
\hline & & & & & \\
\hline & MD, PA, SM, UC & 86. & Lacmellea peruviana (Van Heurck \& Müll. & 102. & Ilex vill \\
\hline 69. & Guatteria & Arg.) & Markgr. & & $000 \mathrm{~m}$. Endémico* \\
\hline & $50 \mathrm{~m} . * \wedge$ & & $800-1250 \mathrm{~m} .{ }^{* \wedge}$ & & CUZ, DAV, \\
\hline & $\mathrm{K}$. & & 1. 1873 (CUZ, F, USM, & & FU) \\
\hline & & & & & \\
\hline & PA, & & AN & ARA & \\
\hline 70. & apoensis Chatrou & 87. & btophylla A.S. Rao & 103. & Dendropanax arboreus (L.) Decne. \& Planch. \\
\hline & & & & & $1000-1500 n$ \\
\hline & $\begin{array}{l}\text { W. Farfan, et al. } 2289 \text { (CUZ, F, USM, } \\
\text { WFU) }\end{array}$ & & $\begin{array}{l}\text { K. Garcia, et al. } 1236 \text { (CUZ, F, USM, } \\
\text { WFU) }\end{array}$ & & $\begin{array}{l}\text { K. Garcia, et al. } 317 \text { (CUZ, F, USM, } \\
\text { WFU) }\end{array}$ \\
\hline & $\mathrm{AM}$ & & $\mathrm{CU}$ & & $\mathrm{AM}$ \\
\hline 71. & Porcelia ponderosa (Rusby) Rusby & 88. & $\begin{array}{l}\text { Rauvolfia sprucei Müll. Arg. } \\
1000-1500 \mathrm{~m} .\end{array}$ & & Dendropanax cuneatus (DC.) Decne. \& \\
\hline & $\begin{array}{l}1000 \\
\text { W. Fa }\end{array}$ & & 3949 (CUZ, F, USM, & & \\
\hline & & & & & 01 (CUZ, F, USM, \\
\hline & & & AM, CU, HU, LO, MD, PA, SM & & \\
\hline 72. & Rollinia andicola Maas \& Westra & AQU & t. \& J. Presl & & HU, LO, 1 \\
\hline & & 89. & Ilex aggregata (Ruiz \& Pav.) Loes. & 105. & Dendropanax \\
\hline & W. Farfan, et al. 1065 (CUZ, F, USM, & & $1800-2250$ m. $* \wedge$ & & $1500-2250$ m. \& \\
\hline & WFU) & & et al. 1113 (CUZ, MO, & & W. Farfan, et al. 1017 (CUZ, DAV, \\
\hline & AM, CA, PA, PI, SM & & & & HUT, MO, USM, WFU) \\
\hline 73. & Rollinia cuspidata Mart. & & HU, PA & 106. & Dendropanax sp. 5 (4748WFR) \\
\hline & $1250 \mathrm{~m} . \wedge$ & 90. & Ilex biserrulata Loes. & & \\
\hline & W. Farfan, et al. 4096 (CUZ, F, USM, & & $2750-3000$ m. * & & W. Farfan, et al. 4748 (MOL, WFU) \\
\hline
\end{tabular}


Tabla 1. Lista de especies arbóreas y afines en los bosques montanos del sureste peruano.

\begin{tabular}{|c|c|c|}
\hline 107. Dendropanax umbellatus (Ruiz \& Pav.) & W. Huaraca, et al. 158 (CUZ, DAV, & AM, CA, CU, HU, PA \\
\hline Decne. \& Planch. & & 141. Pentacalia sp. 1 (37ANQ) \\
\hline $1000-1250 \mathrm{~m} . \wedge$ & 124. Schefflera sprucei Harms & $3537 \mathrm{~m}$ \\
\hline K. Garcia, et al. 328 (CUZ, MO, & $850 \mathrm{~m}$ & A. Nina, et al. 37 (CUZ) \\
\hline USM) & W. Farfan, et al. 5497 (USM) & 142. Piptocarpha lechleri (Sch. Bip.) Baker \\
\hline AM, CU, HU, LO, PA, SM & AM, CU, LO, MD, PA, PU, SM, UC & $1500 \mathrm{~m} . \wedge$ \\
\hline 108. Dendropanax weberbaueri (Harms) Harms & ARECACEAE Bercht. \& J. Presl & K. Garcia, et al. 1245 (F, USM) \\
\hline $1500-1750 \mathrm{~m}$. Endémico ${ }^{\wedge}$ & 125. Bactris setulosa H. Karst. & AM, CU, JU, MD, UC \\
\hline M. N. Raurau, et al. 1429 (MOL) & $1500 \mathrm{~m} . * \wedge$ & 143. Piptocarpha poeppigiana (DC.) Baker \\
\hline $\mathrm{CU}, \mathrm{HU}, \mathrm{LO}$ & W. Farfan, et al. 2750 (USM, WFU) & $800 \mathrm{~m}$ \\
\hline 109. Dendropanax williamsii (Harms) Harms & AM, CA, PA, SM & W. Farfan, et al. 1391 (CUZ, F, USM) \\
\hline $1500-1750 \mathrm{~m}$. Endémico $^{\wedge}$ & 126. Ceroxylon sp. 1 (1170WFR) & AM, CU, HU, JU, LO, MD, PA, SM, \\
\hline K. Garcia, et al. 425 (CUZ, F, USM, & $2000 \mathrm{~m}$ & $\mathrm{UC}$ \\
\hline WFU) & W. Farfan, et al. 1170 (CUZ, MO, & BETULACEAE Gray \\
\hline CU, PA, SM & USM) & 144. Alnus acuminata Kunth \\
\hline 110. Oreopanax capitatus (Jacq.) Decne. \& & 127. Dictyocaryum lamarckianum (Mart.) $\mathrm{H}$. & $2250-2500 \mathrm{~m}$ \\
\hline Planch. & Wendl. & W. Farfan S.N. (CUZ) \\
\hline $1750-2250 \mathrm{~m}$ & $1250-1800 \mathrm{~m} .{ }^{*}$ & AM, AN, AP, CA, CU, HU, JU, LL, \\
\hline W. Farfan, et al. 1149 (CUZ, F, USM, & W. Farfan, et al. 1038 (CUZ) & LI, PA, PI, PU, TA \\
\hline WFU) & PA, MD, SM & BIGNONIACEAE Juss. \\
\hline CU, AM, JU, SM, PA, LO & 128. Euterpe precatoria Mart. & 145. Jacaranda copaia (Aubl.) D. Don \\
\hline 111. Oreopanax kuntzei Harms & $800-1500 \mathrm{~m} .{ }^{*}$ & $800 \mathrm{~m} . \wedge$ \\
\hline $3450 \mathrm{~m} . * * * \wedge$ & W. Farfan, et al. 1311 (CUZ, F) & W. Farfan, et al. 1554A (CUZ, F, \\
\hline W. Farfan, et al. 856 (CUZ, MO, & AM, CA, LO, JU, MD, PA, SM, UC & USM) \\
\hline USM, WFU) & 129. Geonoma undata Klotzsch & AM, CU, HU, LO, MD, PA, SM, UC \\
\hline 112. Oreopanax microflorous Borchs. & $3000 \mathrm{~m} . \wedge$ & 146. Jacaranda glabra (A. DC.) Bureau \& K. \\
\hline $2250-3000 \mathrm{~m} .{ }^{* \wedge}$ & K. Garcia, et al. 1515 (CUZ, F, USM) & Schum. \\
\hline W. Farfan, et al. 939 (CUZ, MO, & AM, CA, CU, HU, MD, PA, SM & $1000-1500 \mathrm{~m}$ \\
\hline USM) & 130. Iriartea deltoidea Ruiz \& Pav. & K. Garcia, et al. 1268 (CUZ, F, USM, \\
\hline CA & $1000-1500 \mathrm{~m}$ & WFU) \\
\hline 113. Oreopanax ruizii Decne. \& Planch. ex & W. Farfan, et al. 2351 (CUZ, F, USM) & AM, AY, CU, HU, LO, MD, PA, PU, \\
\hline Harms & AM, CU, HU, JU, LO, MD, PA, SM, & SM, UC \\
\hline $3625 \mathrm{~m}$ & $\mathrm{UC}$ & BORAGINACEAE Juss. \\
\hline A. Nina, et al. 75 (CUZ) & 131. Socratea exorrhiza (Mart.) H. Wendl. & 147. Cordia mexiana I.M. Johnst. \\
\hline $\mathrm{CU}$ & $1000-1250 \mathrm{~m} \cdot{ }^{* \wedge}$ & $1250 \mathrm{~m} \cdot{ }^{* \wedge}$ \\
\hline 114. Oreopanax sp. 1 (29ANQ) & W. Farfan, et al. 1955 (CUZ, F) & W. Farfan, et al. 2328 (CUZ, F, USM, \\
\hline $3537 \mathrm{~m}$ & AM, JU, LO, MD, PA, SM & WFU) \\
\hline A. Nina, et al. 29 (CUZ) & ASTERACEAE Bercht. \& J. Presl & AM, LO, MD, PU, SM \\
\hline 115. Oreopanax sp. 2 (159WHH) & 132. Ageratina sp. 1 (156WHH) & 148. Cordia panamensis L. Riley \\
\hline $2500-3000 \mathrm{~m} . \&$ & $3000 \mathrm{~m}$ & $1500 \mathrm{~m} .{ }^{* * *}$ \\
\hline $\begin{array}{l}\text { W. Huaraca, et al. } 159 \text { (CUZ, MO, } \\
\text { USM) }\end{array}$ & $\begin{array}{l}\text { W. Huaraca, et al. } 156 \text { (CUZ, DAV, } \\
\text { HUT, MO, USM, WFU) }\end{array}$ & $\begin{array}{l}\text { W. Farfan, et al. } 2725 \text { (CUZ, F, USM, } \\
\text { WFU) }\end{array}$ \\
\hline 116. Oreopanax sp. 3 (2102WFR) & 133. Ageratina sp. 2 (4609WFR) & 149. Cordia scabrifolia A. DC. \\
\hline $1500 \mathrm{~m}$ & $3000 \mathrm{~m}$ & $1000 \mathrm{~m} . * \wedge$ \\
\hline W. Farfan, et al. 2102 (CUZ, F, USM, & W. Farfan, et al. 4609 (MOL, WFU) & W. Farfan, et al. 1497 (CUZ, F, USM) \\
\hline WFU) & 134. Baccharis oblongifolia (Ruiz \& Pav.) Pers. & $\mathrm{LO}, \mathrm{MD}, \mathrm{SM}$ \\
\hline 117. Oreopanax sp. 4 (4688WFR) & $3000 \mathrm{~m} .{ }^{*}$ & 150. Cordia sp. 5 (1484AWFR) \\
\hline $2890 \mathrm{~m}$ & W. Farfan, et al. 1249 (CUZ, F, USM) & $1000 \mathrm{~m}$ \\
\hline W. Farfan, et al. 4688 (MOL, WFU) & AM, CA, PA & W. Farfan, et al. 1484 (CUZ, F, USM) \\
\hline 118. Schefflera acuminata (Ruiz \& Pav.) Harms & 135. Baccharis salicifolia (Ruiz \& Pav.) Pers. & 151. Cordia sp. 6 \\
\hline $1750 \mathrm{~m}$ & $2890 \mathrm{~m}$ & $1500 \mathrm{~m}$ \\
\hline W. Farfan, et al. 4919 (MOL) & W. Farfan, S.N. (CUZ) & W. Farfan S.N. (CUZ) \\
\hline $\mathrm{CA}, \mathrm{CU}, \mathrm{HU}, \mathrm{JU}, \mathrm{PA}$ & $\mathrm{AM}, \mathrm{AN}, \mathrm{AP}, \mathrm{AR}, \mathrm{CA}, \mathrm{CU}, \mathrm{HU}, \mathrm{LL}$ & 152. Cordia trachyphylla Mart. \\
\hline 119. Schefflera allocotantha (Harms) Frodin & LI, LA, MD, PA, SM, UC & $800-1000 \mathrm{~m} \cdot{ }^{* \wedge}$ \\
\hline $1800-3250 \mathrm{~m} .^{* * * \wedge}$ & 136. Barnadesia caryophylla (Vell.) S.F. Blake & W. Farfan, et al. 3293 (CUZ, F, USM) \\
\hline A. R. Davila, et al. 36 (CUZ, DAV, & $1250 \mathrm{~m} .{ }^{*}$ & LO \\
\hline HUT, MO, USM, WFU) & W. Farfan, et al. 1928 (CUZ, F, USM) & 153. Cordia ucayaliensis (I.M. Johnst.) I.M. Johnst. \\
\hline 120. Schefflera inambarica Harms & AM, JU, PA, PU & $850 \mathrm{~m}$ \\
\hline $2000-2250 \mathrm{~m}$ & 137. Gynoxys nitida Muschl. & W. Farfan, et al. 5500 (CUZ) \\
\hline W. Farfan, et al. 3122 (CUZ, F, USM, & $3537-3625 \mathrm{~m}$ & $\mathrm{AM}, \mathrm{CA}, \mathrm{CU}, \mathrm{HU}, \mathrm{LO}, \mathrm{MD}, \mathrm{PA}$ \\
\hline WFU) & A. Nina, et al. 120 (CUZ) & PU, SM, UC \\
\hline CU, JU, PA, PU & AN, AY, CU, LI, JU, PA & BRUNELLIACEAE Engl. \\
\hline 121. Schefflera morototoni (Aubl.) Maguire, & 138. Nordenstamia repanda (Wedd.) Lundin & 154. Brunellia boliviana Britton ex Rusby \\
\hline Steyerm. \& Frodin & $3250-3450 \mathrm{~m}$ & $3250-3450 \mathrm{~m} . \wedge$ \\
\hline $1250 \mathrm{~m} . \wedge$ & W. Farfan, et al. 858 (CUZ, DAV, & W. Farfan, et al. 829 (CUZ, MO, \\
\hline K. Garcia, et al. 745 (CUZ, F, USM, & HUT, MO, USM, WFU) & USM, WFU) \\
\hline WFU) & $\mathrm{CU}, \mathrm{PU}$ & $\mathrm{CU}$ \\
\hline AM, CU, HU, JU, LO, MD, SM, UC & 139. Nordenstamia sp. 1 (817WFR) & 155. Brunellia brunnea J.F. Macbr. \\
\hline 122. Schefflera patula (Rusby) Harms & $3250-3450 \mathrm{~m}$ & $2000 \mathrm{~m} . \wedge$ \\
\hline $1800-2250 \mathrm{~m}$ & W. Farfan, et al. 817 (CUZ, USM, & W. Farfan, et al. 1181 (CUZ, MO, \\
\hline W. Farfan, et al. 3338 (CUZ, F, USM, & WFU) & USM, WFU) \\
\hline WFU) & 140. Pentacalia oronocensis (DC.) Cuatrec. & CU, JU, SM \\
\hline AM, CA, CU, CU, LO, PA, PU, SM & $3000-3450 \mathrm{~m}$ & 156. Brunellia cuzcoensis Cuatrec. \\
\hline 123. Schefflera sp. 1 (158WHH) & W. Huaraca, et al. 153 (CUZ, DAV, & $3000 \mathrm{~m}$. Endémico \\
\hline $3000-3250 \mathrm{~m} . \&$ & HUT, MO, USM, WFU) & J. E. Silva, et al. 638 (CUZ, DAV, \\
\hline
\end{tabular}


Tabla 1. Lista de especies arbóreas y afines en los bosques montanos del sureste peruano.

\begin{tabular}{|c|c|c|c|}
\hline & HUT, MO, USM, WFU) & $850 \mathrm{~m} . * \wedge$ & 188. Dendrobangia boliviana Rusby \\
\hline & $\mathrm{CU}$ & W. Farfan, et al. 5523 (CUZ) & $800 \mathrm{~m} .{ }^{*}$ \\
\hline \multirow[t]{5}{*}{157.} & Brunellia dulcis J.F. Macbr. & AM, LO, MD, SM, UC & W. Farfan, et al. 1319 (CUZ, F, USM, \\
\hline & $2250 \mathrm{~m}$. Endémico* & 173. Protium sp. 1 (1040WFR) & WFU) \\
\hline & W. Farfan, et al. 1082 (CUZ, MO, & $1750-1800 \mathrm{~m}$ & $\mathrm{AM}, \mathrm{LO}, \mathrm{MD}, \mathrm{PA}, \mathrm{SM}$ \\
\hline & USM, WFU) & W. Farfan, et al. 1040 (CUZ, MO, & 189. Dendrobangia sp. 1 (1905WFR) \\
\hline & CA, PA & USM, WFU) & $1250 \mathrm{~m}$ \\
\hline \multirow[t]{4}{*}{158.} & Brunellia inermis Ruiz \& Pav. & 174. Protium sp. 2 (973KGC) & W. Farfan, et al. 1905 (CUZ, F, USM) \\
\hline & $2500-3450 \mathrm{~m} .{ }^{* \wedge}$ & $1750 \mathrm{~m}$ & CARICACEAE Dumort. \\
\hline & $\begin{array}{l}\text { W. Farfan, et al. } 843 \text { (CUZ, MO, } \\
\text { USM, WFU) }\end{array}$ & $\begin{array}{l}\text { K. Garcia, et al. } 973 \text { (CUZ, USM, } \\
\text { WFU) }\end{array}$ & $\begin{array}{l}\text { 190. Jacaratia digitata (Poepp. \& Endl.) Solms } \\
1250-1500 \mathrm{~m} .\end{array}$ \\
\hline & CA, HU, JU, PA & 175. Protium spruceanum (Benth.) Engl. & W. Farfan, et al. 1776 (CUZ, F, USM) \\
\hline \multirow[t]{4}{*}{159.} & Brunellia littlei Cuatrec. & $1500 \mathrm{~m} . * \wedge$ & AM, CU, JU, HU, LO, MD, PA, PI, \\
\hline & $2000 \mathrm{~m} .{ }^{* * *}$ & W. Farfan, et al. 4793 (MOL, WFU) & $\mathrm{SM}$ \\
\hline & W. Farfan, et al. 1130 (CUZ, MO, & $\mathrm{AM}, \mathrm{LO}, \mathrm{MD}$ & CARYOCARACEAE Voigt \\
\hline & USM, WFU) & 176. Tetragastris panamensis (Engl.) Kuntze & 191. Anthodiscus pern \\
\hline \multirow[t]{5}{*}{160.} & Brunellia stenoptera Diels & $850 \mathrm{~m} .{ }^{*}$ & 800 m. * $^{*}$ \\
\hline & $1500 \mathrm{~m} . * \wedge$ & W. Farfan, et al. 5507A (USM) & W. Farfan, et al. 1292 (CUZ, MO) \\
\hline & K. Garcia, et al. 1134 (CUZ, F, USM, & AM, JU, LO, MD, PA, SM, UC & AM, HU, LO, MD, PA, SM \\
\hline & WFU) & Trattinnickia boliviana (Swart) D.C. Daly & 192. Caryocar amygdaliforme Ruiz \& Pav. ex G. \\
\hline & JU & $800 \mathrm{~m} .{ }^{*} \wedge$ & Don \\
\hline \multirow[t]{5}{*}{161.} & Brunellia weberbaueri Loes. & W. Farfan, et al. 2014 (CUZ, F, USM, & $800-1500 \mathrm{~m} \cdot{ }^{* \wedge}$ \\
\hline & $2750 \mathrm{~m}$. Endémico $^{\wedge}$ & WFU) & W. Farfan, et al. 2556 (CUZ, F, USM, \\
\hline & W. Farfan, et al. 892 (CUZ, DAV, & AM, PA & WFU) \\
\hline & HUT, MO, USM, WFU) & 178. Trattinnickia burserifolia Mart. & LO, MD, PA, SM \\
\hline & $\mathrm{CU}, \mathrm{PA}$ & & 193. Caryocar glabrum Pers. \\
\hline \multicolumn{2}{|c|}{ BURSERACEAE Kunth } & W. Farfan, et al. 1293 (CUZ, MO, & $800-1750 \mathrm{~m} .^{*}$ \\
\hline \multirow[t]{4}{*}{162.} & Dacryodes peruviana (Loes.) H.J. Lam & USM, WFU) & K. Garcia, et al. 281 (CUZ, MO, \\
\hline & $850 \mathrm{~m} .^{*}$ & LO, MD & USN \\
\hline & W. Farfan, et al. 5507 (CUZ) & 179. Trattinnickia glaziovii Swart & AM, HU, JU, LL, LO, PA, SM \\
\hline & $\mathrm{D}, \mathrm{PA}, \mathrm{SM}$ & $800 \mathrm{~m} .{ }^{*} \wedge$ & 194. Caryocar pallidum A.C. Sm. \\
\hline \multirow[t]{4}{*}{163.} & Dacryodes sp. 1 (621KGC) & W. Farfan, et al. 1260 (CUZ, WFU) & $1500-1750 \mathrm{~m} . \wedge$ \\
\hline & $1500 \mathrm{~m}$ & $\mathrm{LO}, \mathrm{MD}, \mathrm{PA}$ & K. Garcia, et al. 963 (CUZ, MO, \\
\hline & K. Garcia, et al. 621 (CUZ, F, USM, & BUXACEAE Du & \\
\hline & WFU) & 180. Styloceras brokawii A.H. Gentry \& R.B. & $\mathrm{CU}, \mathrm{LO}, \mathrm{MD}, \mathrm{PU}$ \\
\hline \multirow[t]{5}{*}{164.} & Protium altsonii Sandwith & Foster & CELASTRACEAE R. Br. \\
\hline & $800 \mathrm{~m} .{ }^{*}$ & $1500 \mathrm{~m} \cdot{ }^{* \wedge}$ & 195. Cheiloclinium cognatum (Miers) A.C. Sm. \\
\hline & W. Farfan, et al. 1285 (CUZ, MO, & K. Garcia, et al. 1271 (CUZ, F, USM, & $1500 \mathrm{~m} .{ }^{*}$ \\
\hline & USM, WFU) & WFU) & arcia, et al. 619 (CUZ, F, USM, \\
\hline & LO, PA, SM & $\mathrm{MD}$ & WFU) \\
\hline \multirow[t]{5}{*}{165.} & Protium decandrum (Aubl.) Marchand & CALOPHYLLACEAE J. Agardh & AM, HU, JU, LI, LO, MD, PA, SM, \\
\hline & $800-1500 \mathrm{~m} \cdot{ }^{* \wedge}$ & brasiliense Cambess. & $\mathrm{UC}$ \\
\hline & W. Farfan, et al. 1278 (CUZ, MO, & $850 \mathrm{~m} .{ }^{*}$ & 196. Maytenus ebenifolia Reissek \\
\hline & USM, WFU) & W. Farfan, et al. 5526 (CUZ) & \\
\hline & LO, AM & AM, LO, MD, PA, SM & K. Garcia, et al. 756 (CUZ, F, USM, \\
\hline 166. & Protium glabrescens Swart & 182. Marila laxiflora Rusby & WFU) \\
\hline & $1500-1750 \mathrm{~m} .{ }^{*} \wedge$ & $1250 \mathrm{~m} . \wedge$ & CU, JU, HU \\
\hline & W. Farfan, et al. 4171 (MOL, WFU) & W. Farfan, et al. 4150 (MOL, WFU) & 197. Maytenus macrocarpa (Ruiz \& Pav.) Briq. \\
\hline & $\mathrm{AM}, \mathrm{LO}, \mathrm{MD}, \mathrm{PA}, \mathrm{SM}$ & AM, CU, HU, LO, MD, PA, PU, & $1250 \mathrm{~m} .^{*}$ \\
\hline 167. & Protium hebetatum D.C. Daly & SM & W. Farfan, et al. 3391A (CUZ, USM) \\
\hline & $800 \mathrm{~m}$ & 183. Marila sp. 1 (5532WFR) & AM, HU, LO, MD, PA, SM, TU \\
\hline & W. Farfan, et al. 1232 (CUZ, MO, & $850 \mathrm{~m}$ & 198. Maytenus sp. 1 (5536WFR) \\
\hline & USM, WFU) & W. Farfan, et al. 5532 (CUZ) & 850 \\
\hline & LO, PA & CAMPANULACEAE Juss. & W. Farfan, et al. 5536 (CUZ) \\
\hline 168. & Protium montanum Swart & 184. Centropogon sp. 1 & 199. Maytenus sp. 3 (3391WFR) \\
\hline & $1250-1800 \mathrm{~m} \cdot \wedge$ & $3537 \mathrm{~m}$ & $1250 \mathrm{~m}$ \\
\hline & W. Farfan, et al. 1014 (CUZ, MO, & W. Farfan S.N. (CUZ) & W. Farfan, et al. 3391 (CUZ, F, USM) \\
\hline & USM, WFU) & 185. Siphocampylus vatkeanus Zahlbr. & 200. Salacia sp. 1 (4541WFR) \\
\hline & $\mathrm{CU}$ & $3625 \mathrm{~m} . \wedge$ & $2000 \mathrm{~m}$ \\
\hline 169. & Protium opacum cf. Swart & A. Nina, et al. 81 (CUZ) & W. Farfan, et al. 4541 (MOL, WFU) \\
\hline & $800 \mathrm{~m} . \wedge$ & CU, PU & CHLORANTHACEAE R. Br. ex Sims \\
\hline & W. Farfan, et al. 1400 (CUZ, F, USM, & CANNABACEAE Martinov & 201. Hedyosmum anisodorum Todzia \\
\hline & WFU) & 186. Trema micrantha (L.) Blume & $2250 \mathrm{~m}$ \\
\hline & AM, CU, HU, LO, MD, PA, PU, SM & $1250-1500 \mathrm{~m}$ & W. Farfan, et al. 1148 (CUZ, MO, \\
\hline 170. & Protium plagiocarpium Benoist & W. Farfan, et al. 1589 (CUZ, F, USM, & USM, WFU) \\
\hline & $800 \mathrm{~m} . * \wedge$ & WFU) & $\mathrm{CA}, \mathrm{CU}, \mathrm{PA}$ \\
\hline & W. Farfan, et al. 1394 (CUZ, MO, & $\mathrm{AM}, \mathrm{AP}, \mathrm{CA}, \mathrm{CU}, \mathrm{HU}, \mathrm{JU}, \mathrm{LO}, \mathrm{MD}$, & 202. Hedyosmum cuatrecazanum Occhioni \\
\hline & USM, WFU) & & \\
\hline & $\mathrm{LO}, \mathrm{MD}$ & CARDIOPTERIDACEAE Blume & K. Garcia, et al. 929 (CUZ, F, USM, \\
\hline 171. & Protium rhynchophyllum (Rusby) D.C. Daly & 187. Citronella incarum (J.F. Macbr.) R.A. Howard & WFU) \\
\hline & $1500-1750 \mathrm{~m}$ & $1500 \mathrm{~m}$ & CA, CU, HU, PA, SM \\
\hline & K. Garcia, et al. 1665 (CUZ, F, USM, & K. Garcia, et al. 1284 (CUZ, F, USM, & 203. Hedyosmum goudotianum Solms \\
\hline & WFU) & WFU) & $1800-3250 \mathrm{~m} .{ }^{*}$ \\
\hline & LO, MD & AM, CA, CU, HU, LO, MD, PA, PI, & W. Farfan, et al. 577 (CUZ, DAV, \\
\hline 172. & Protium sagotianum Marchand & PU, SM, UC & HUT, MO, USM, WFU) \\
\hline
\end{tabular}


Tabla 1. Lista de especies arbóreas y afines en los bosques montanos del sureste peruano.

\begin{tabular}{|c|c|c|c|}
\hline & CA, JU, MD, PA, SM & LO, MD, PA & HUT, MO, USM, WFU) \\
\hline \multirow[t]{5}{*}{204.} & Hedyosmum maximum (Kuntze) K. Schum. & 221. Parinari parilis J.F. Macbr. & 237. Clusia sp. 10 (755MRQ) \\
\hline & $3000-3250 \mathrm{~m} . \wedge$ & $1500-1750 \mathrm{~m} . \wedge$ & $1750 \mathrm{~m}$ \\
\hline & M. Mamami, et al. 333 (CUZ, MO, & K. Garcia, et al. 998 (CUZ, F, USM, & W. Farfan, et al. 755 (CUZ, F) \\
\hline & USM, WFU) & WFU) & 238. Clusia sp. 13 (3751WFR) \\
\hline & $\mathrm{CU}$ & CU, LO, MD, PA, SM & $1750 \mathrm{~m}$ \\
\hline \multirow[t]{5}{*}{205.} & Hedyosmum peruvianum Todzia & CLETHRACEAE Klotzsch & W. Farfan, et al. 3751 (CUZ, USM, \\
\hline & $1750-2890 \mathrm{~m}$. Endémico* ${ }^{\wedge}$ & 222. Clethra castaneifolia Meisn. & WFU) \\
\hline & W. Farfan, et al. 918 (CUZ, DAV, & $2890 \mathrm{~m}$ & Clusia sp. 14 (4955WFR) \\
\hline & HUT, MO, USM, WFU) & W. Farfan, et al. 4681 (MOL, WFU) & \\
\hline & CA, HU, PA, SM & AM, CA, CU, SM, JU, LA, PA, SM & W. Farfan, et al. 4955 (MOL, WFU) \\
\hline \multirow{6}{*}{$\begin{array}{l}206 . \\
\text { Don }\end{array}$} & Hedyosmum racemosum (Ruiz \& Pav.) G. & 223. Clethra cuneata Rusby & Clusia sp. 4 (3101)WFR) \\
\hline & & $2890-3537 \mathrm{~m}$ & \\
\hline & $1750-2000 \mathrm{~m}$ & W. Farfan, et al. 813 (CUZ, DAV, & W. Farfan, et al. 3101 (CUZ, F, USM) \\
\hline & W. Farfan, et al. 3339 (CUZ, F, USM, & HUT, MO, USM, WFU) & 241. Clusia sp. 6 (1013WFR) \\
\hline & WFU) & AM, CA, CU, JU, PA, SM & \\
\hline & $\begin{array}{l}\text { AM, CA, CU, HU, JU, LA, MD, PA, } \\
\text { SM }\end{array}$ & $\begin{array}{l}\text { 224. Clethra ferruginea (Ruiz \& Pav.) Link ex } \\
\text { Spreng. }\end{array}$ & $\begin{array}{l}\text { W. Farfan, et al. } 1013 \text { (CUZ, MO, } \\
\text { USM, WFU) }\end{array}$ \\
\hline \multirow[t]{5}{*}{207.} & Hedyosmum scabrum (Ruiz \& Pav.) Solms & $3000-3450 \mathrm{~m}$ & Clusia sp. 7 (4536WFR) \\
\hline & $2890-3450 \mathrm{~m} . \wedge$ & W. Farfan, et al. 852 (CUZ, MO, & $2000 \mathrm{~m}$ \\
\hline & n, et al. 850 (CUZ, DAV, & USM) & W. Farfan, et al. 4536 (MOL, WFU) \\
\hline & HUT, MO, USM, WFU) & $\mathrm{U}, \mathrm{HU}, \mathrm{SM}$ & a Planch. \& Triana \\
\hline & JU, PA, PI, SM & 225. Clethra obovata (Ruiz \& Pav.) G. Don & $\mathrm{m} \cdot \wedge$ \\
\hline \multirow[t]{3}{*}{208.} & Hedyosmum sp. 1 (57ANQ) & $500 \mathrm{~m}$ & al. 597 (CUZ, MO) \\
\hline & $3537 \mathrm{~m}$. & l. 1974 (CUZ, F, USM) & PA, PI \\
\hline & , et al. 57 (CUZ) & M & lanch. \& Triana \\
\hline \multirow[t]{3}{*}{209.} & Hedyosmum sp. 3 (4510WFR) & 226. Clethra revoluta (Ruiz \& Pav.) Spreng. & $2250 \mathrm{~m} \cdot \wedge$ \\
\hline & $2250 \mathrm{~m}$ & $1750-2750 \mathrm{~m}$ & W. Farfan, et al. 3106 (CUZ, F, USM, \\
\hline & W. Farfan, et al. 4510 (MOL, WFU) & W. Farfan, et al. 967 (CUZ, MO, & \\
\hline \multirow[t]{5}{*}{210.} & Hedyosmum translucidum Cuatrec. & USM, WFU) & CU, PA, PU, SM \\
\hline & $90 \mathrm{~m} .{ }^{*}$ & CU, PA, SM & Clusia trochifor \\
\hline & W. Farfan, et al. 1030 (CUZ, DAV, & 227. Clethra scabra Pers. & \\
\hline & HUT, MO, USM, WFU) & $1250 \mathrm{~m}$ & et al. 4682 (MOL, WFU) \\
\hline & AM, CA, PA, PI & W. Farfan, et al. 1961 (CUZ, F, USM, & $\mathrm{PA}, \mathrm{SM}$ \\
\hline \multicolumn{2}{|c|}{ CHRYSOBALANACEAE R. Br. } & WFU) & 246. Garcinia madruno (Kunth) Hammel \\
\hline \multicolumn{2}{|c|}{ 211. Couepia bernardii Prance } & JU, CU, AM, PA, CA & $500 \mathrm{~m} . * \wedge$ \\
\hline & $850 \mathrm{~m} . * \wedge$ & 228. Clethra sp. 1 (584WFR) & K. Garcia, et al. 565 (CUZ, F, USM, \\
\hline & W. Farfan, et al. 5544 (CUZ) & $2750-3537 \mathrm{~m}$ & WFU) \\
\hline & LO & 1. 584 (CUZ, DAV, & $\mathrm{AN}$ \\
\hline \multirow[t]{3}{*}{212.} & Couepia sp. 1 (5541WFR) & MO, USM, WFU) & sis (Mart.) Planch. \& Triana \\
\hline & $850 \mathrm{~m}$ & 229. Clethra sp. 2 (876WFR) & $\mathrm{m} . * \wedge$ \\
\hline & W. Farfan, et al. 5541 (CUZ) & 275 & an, et al. 1420 (CUZ, F, USM) \\
\hline 213. & Hirtella sp. 1 (1378AWFR) & W. Farfan, et al. 876 (CUZ, F, USM) & 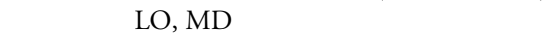 \\
\hline & $800 \mathrm{~m}$ & 230. Clethra sp. 3 (1119WFR) & Symphonia globulifera L. f. \\
\hline & W. Farfan, et al. 1378A (CUZ, F) & $2000 \mathrm{~m}$ & \\
\hline 214. & Hirtella triandra Sw. & W. Farfan, et al. 1119 (CUZ) & W. Farfan, et al. 2306 (CUZ, MO, \\
\hline & $1250 \mathrm{~m}$ & CLUSIACEAE Lindl. & \\
\hline & K. Garcia, et al. 664 (USM) & 231. Chrysochlamys ulei Engl. & AM, CU, HU, JU, LO, MD, PA, PU, \\
\hline & LO, MD, SM, UC & $1250-1500 n$ & \\
\hline 215. & Licania kunthiana Hook. f. & W. Farfan, et al. 2722 (CUZ, F, USM, & Tovomita sp. 1(1048WFR) \\
\hline & $800 \mathrm{~m} .{ }^{*}$ & WFU) & \\
\hline & W. Farfan, et al. 1300 (CUZ, MO, & $\mathrm{MD}$ & et al. 1048 (CUZ, MO, \\
\hline & USM, & 232. Clusia alata $\mathrm{P}$ & \\
\hline & AM, PU & $2750-3450 \mathrm{~m}$ & Tovomita weddelliana Planch. \& Triana \\
\hline 216. & Licania macrocarpa Cuatrec. & W. Farfan, et al. 812 (CUZ, MO, & $800-2000 \mathrm{~m} \cdot{ }^{* \wedge}$ \\
\hline & $1500-1750 \mathrm{~m} .{ }^{* \wedge}$ & USM) & W. Farfan, et al. 1158 (CUZ, MO, \\
\hline & K. Garcia, et al. 594 (CUZ, F, USM, & LO, CU, PA, SM & USM, WFU) \\
\hline & WFU) & 233. Clusia ducuoides Engl. & AM, LO, MD, PA, PU, SM \\
\hline & LO, HU, PA & $1800-2250$ m. * & CUNONIACEAE R. Br. \\
\hline 217. & Licania micrantha Miq. & W. Farfan, et al. 3124 (CUZ, F, USM, & 251. Weinmannia auriculata D. Don \\
\hline & & & \\
\hline & W. Farfan, et al. 1397 (CUZ, F, USM, & AM, CA, HU, PA, PI, SM & W. Farfan, et al. 626 (CUZ, DAV, \\
\hline & WFU) & 234. Clusia elliptica Kunth & HUT, MO, USM, WFU) \\
\hline & AM, HU, LO, PA & $1750-2890 \mathrm{~m} .^{* \wedge}$ & AM, CA, CU, HU, PA, SM \\
\hline & Licania octandra (Hoffmanns. ex Roem. \& & 1. 1035 (CUZ, DAV, & Weinmannia balbisiana Kunth \\
\hline Schul & It.) Kuntze & HUT, MO, USM, WFU) & $2000 \mathrm{~m} . * \wedge$ \\
\hline & $850 \mathrm{~m} \cdot \wedge$ & $\mathrm{AM}, \mathrm{CA}, \mathrm{PA}, \mathrm{SM}$ & W. Farfan, et al. 1103 (CUZ, HUT, \\
\hline & W. Farfan, et al. 5546 (CUZ) & 235. Clusia pavonii Planch. \& Triana & MO, USM, WFU) \\
\hline & CU, LO, MD, PA, SM, UC & $3450 \mathrm{~m} . \wedge$ & CA, PA, SM \\
\hline 219. & Licania sp. 3 & W. Farfan, et al. 861 (CUZ, DAV, & Weinmannia bangii Rusby \\
\hline & $1750 \mathrm{~m}$ & HUT, MO, USM, WFU) & $2500-3250 \mathrm{~m} . \wedge$ \\
\hline & W. Farfan S.N. (CUZ) & $\mathrm{AM}, \mathrm{CA}, \mathrm{CU}$ & W. Farfan, et al. 630 (CUZ, DAV, \\
\hline 220. & Parinari occidentalis Prance & 236. Clusia sp. 1 (1048WFR) & HUT, MO, USM, WFU) \\
\hline & $850 \mathrm{~m} \cdot{ }^{* \wedge}$ & $2000 \mathrm{~m}$ & $\mathrm{CU}$ \\
\hline & W. Farfan, et al. 5557 (CUZ) & W. Farfan, et al. 1048 (CUZ, DAV, & Weinmannia cochensis Hieron. \\
\hline
\end{tabular}


Tabla 1. Lista de especies arbóreas y afines en los bosques montanos del sureste peruano.

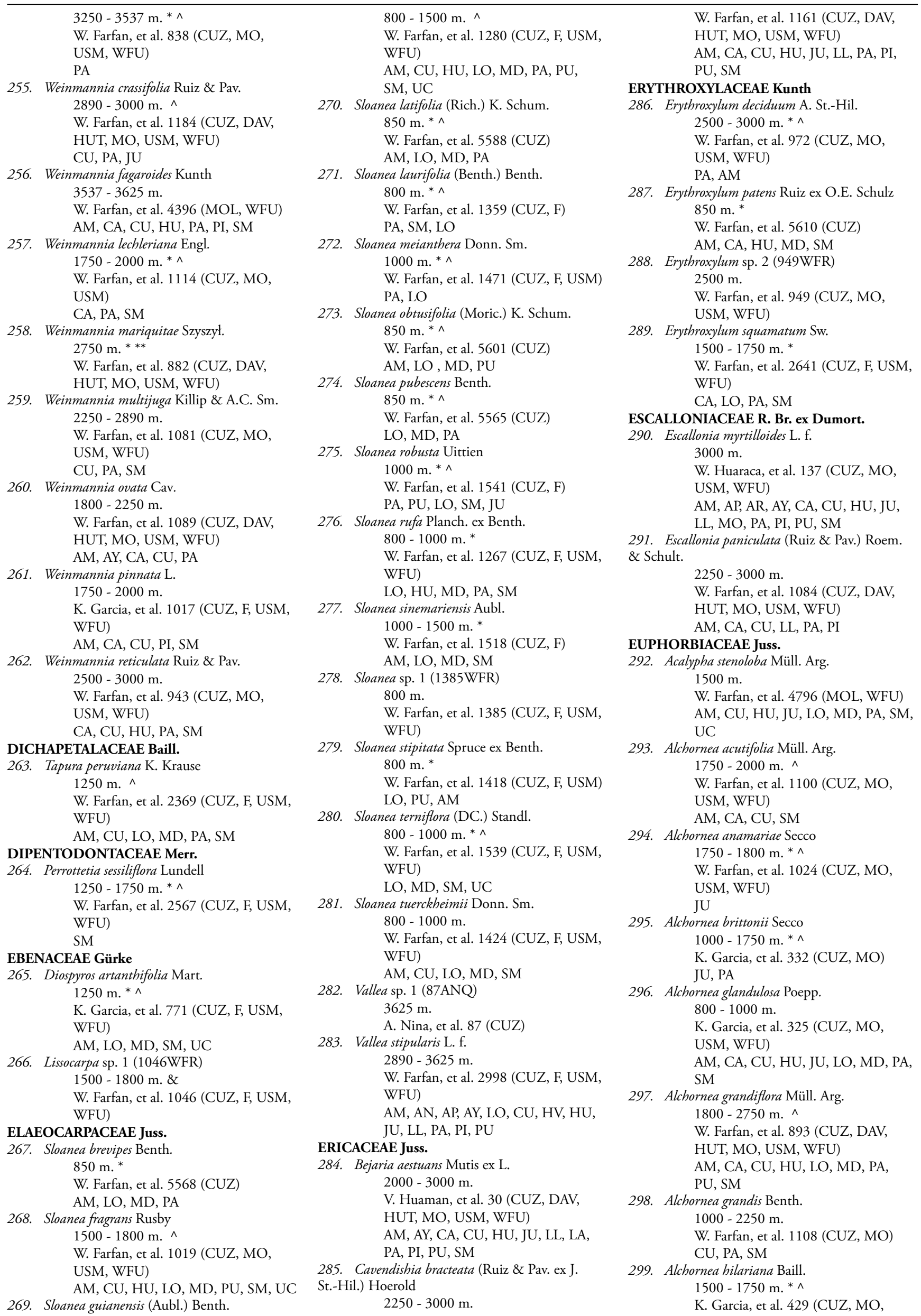


Tabla 1. Lista de especies arbóreas y afines en los bosques montanos del sureste peruano.

\begin{tabular}{|c|c|c|c|c|}
\hline & USM, WFU) & TU, UC & 335. & Inga barbata Benth. \\
\hline & $\mathrm{MD}$ & Sapium marmieri Huber & & $1500 \mathrm{~m} . * \wedge$ \\
\hline 300. & Alchornea latifolia Sw. & $1500 \mathrm{~m}$ & & K. Garcia, et al. 616 (CUZ, F, USM, \\
\hline & $1250-1800 \mathrm{~m} . * \wedge$ & K. Garcia, et al. 592 (CUZ, F, USM, & & WFU) \\
\hline & K. Garcia, et al. 475 (CUZ, MO, & WFU) & & MD \\
\hline & USM, WFU) & AM, CU, HU, LO, MD, PA, SM, UC & 336. & Inga bourgonii (Aubl.) DC. \\
\hline & CA, LO, SM, UC & 320. Sapium sp. 1 (1103KGC) & & $1250-1$ \\
\hline 301. & Alchornea pearcei Britton ex Rusby & $1500 \mathrm{~m}$ & & et al. 1910 (CUZ, F, USM) \\
\hline & $2250 \mathrm{~m} . * \wedge$ & K. Garcia, et al. 1103 (CUZ, F, USM, & & AM, JU, LO, MD, PA, SM \\
\hline & W. Farfan, et al.1146 (CUZ, MO, & WFU) & 337. & Inga capitata Desv. \\
\hline & USM, W & 321. Senefeldera inclinata Müll. Arg. & & \\
\hline & CA, LO, P & $800 \mathrm{~m}$ & & ia, et al. 704 (CUZ, F, USM, \\
\hline 302. & Alchornea sp. 1 & W. Farfan, et al. 1399 (CUZ, F, USM, & & \\
\hline & $1500 \mathrm{~m}$ & WFU) & & AM, CA, HU, LO, MD, PA, SM, UC \\
\hline & K. Garcia, et al. 400 (CUZ, MO) & AM, CU, HU, LO, MD, PU, SM, UC & 338. & Inga chartacea $\mathrm{P}$ \\
\hline 303. & Alchornea sp. 10 (3297AWFR) & 322. Tetrorchidium ru & & \\
\hline & $800 \mathrm{~m}$ & $1250-15$ & & in, et al. 2329 (CUZ, F, USM, \\
\hline & W. Farfan, et al. 3297A (CUZ) & al. 409 (CUZ, F, USM, & & \\
\hline 304. & Alchornea sp. 11 & WFU) & & AM, CU, LO, MD, PA, PU, SM, UC \\
\hline & $1800-20$ & AM, CU, HU, JU, LO, PA, SM & 339. & Inga corda \\
\hline & 087 (CUZ, MO, & FABACEAE Lindl. & & \\
\hline & USM, WFU) & 323. Abarema jupunba (Willd.) Britton \& Killip & & an, et al. 5665 (CUZ) \\
\hline 305. & Alchornea sp. 2 (434KGC) & $800-1000 \mathrm{~m} .^{*}$ & & \\
\hline & $1750 \mathrm{~m}$ & et al. 1490 (CUZ, F, USM, & 340. & Inga densiflora $\mathrm{H}$ \\
\hline & K. Garcia, et al. 434 (CUZ, MO) & WFU) & & \\
\hline 306. & Alchornea sp & $\mathrm{AM}$, & & K. Garcia, et al. 794 (CUZ, F, USM, \\
\hline & $800-$ & Andira inermis (W. Wright) Kunth ex DC. & & 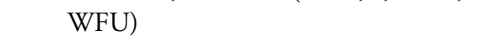 \\
\hline & al. $294(\mathrm{CUZ}, \mathrm{F})$ & $\mathrm{m} . \wedge$ & & CU, HU, LO, MD, SM \\
\hline 307. & Alchornea sp. 6 & , et al. 370 (CUZ, MO, & 341. & Inga ed \\
\hline & $1750 \mathrm{~m}$ & ) & & 1 \\
\hline & 5 (CUZ, MO, USM) & $\mathrm{O}, \mathrm{MD}, \mathrm{PA}, \mathrm{SM}, \mathrm{UC}$ & & 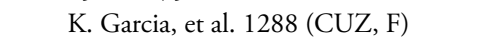 \\
\hline 308. & Alchornea sp. 7 (982KGC) & 325. Bauhinia sp. 1 (5698WFR) & & A, CU, HU, JU, LO, MD, \\
\hline & $1750 \mathrm{~m}$ & $850 \mathrm{~m}$ & & \\
\hline & 2 (CUZ, MO, USM) & 8 (CUZ) & 342. & Inga gracilifolia Ducke \\
\hline 309. & Alchornea sp. 8 (1026KGC) & 326. Cedrelinga cateniformis (Ducke) Ducke & & \\
\hline & $1750 \mathrm{~m}$ & $0 \mathrm{~m} \cdot \wedge$ & & n, et al. 5664 (CUZ) \\
\hline & (CUZ, MO) & arfan, et al. 1327 (CUZ, F, USM, & & \\
\hline 310. & Alchornea sp. 9 & J) & 343. & Inga he \\
\hline & $1500-$ & AM, CU, HU, JU, LO, MD, PA, PU, & & $800 \mathrm{~m} . \wedge$ \\
\hline & K. Gar & SM & & al. 1075 (CUZ, DAV, \\
\hline 311. & Alchornea triplinervia (Spreng.) Müll. Arg. & 327. Cyathostegia mathewsii cf. (Benth.) Schery & & FU) \\
\hline & $800 \mathrm{~m}$. & 100 & & $\mathrm{U}, \mathrm{LO}, \mathrm{MD}, \mathrm{PA}, \mathrm{SM}$ \\
\hline & 34 (CUZ, F, USM) & 1503 (CUZ, F, USM) & 344. & Inga ki \\
\hline & LO, PA, AM, CU, UC, SM & $\mathrm{AM}, \mathrm{AP}$ & & lémico ${ }^{\wedge}$ \\
\hline 312. & Aparisthmium cordatum (A. Juss.) Baill. & 328. Dussia tessmannii Harms & & CUZ, F) \\
\hline & $800 \mathrm{~m} .{ }^{*}$ & & & \\
\hline & al. 283 (CUZ, MO, USM) & al. 2348A (CUZ, F, & 345. & Sw.) Willd. \\
\hline & AM, HU, LO, & ) & & \\
\hline 313. & Croton sp. 1 (656KGC) & AM, CU, LO, MD, PA, SM & & W. Farfan, et al. 2302 (CUZ, F, USM, \\
\hline & $1250 \mathrm{~m}$ & 329. Enterolobium sp. 1 (5686WFR) & & \\
\hline & K. Garcia, et al. 656 (CUZ, F, USM, & $850 \mathrm{~m}$ & & JU, LO, $N$ \\
\hline & WFU) & 5686 (CUZ) & 346. & Inga let \\
\hline 314. & Croton sp. 2 (4588WFR) & 330. Erythrina ulei Harms & & 80 \\
\hline & $1800 \mathrm{~m}$ & 150 & & et al. 1299 (CUZ, MO, \\
\hline & W. Farfan, et al. 4588 (MOL, WFU) & fon 1250 (CUZ E USM & & \\
\hline 315. & Hevea guianensis Aubl. & WFU) & & $\mathrm{AM}, \mathrm{CU}, \mathrm{I}$ \\
\hline & $800 \mathrm{~m}$. & AM, CU, HU, JU, LO, MD, PA, SM, & 347. & Inga macrophylla Humb. \& Bonpl. ex Willd. \\
\hline & K. Garcia, et al. 280 (CUZ, F, USM, & UC & & \\
\hline & WFU) & 331. Hymenaea oblongifolia Huber & & 1. 728 (CUZ, F, USM, \\
\hline & AM, CU, HU, JU, LO, MD, PA, PU, & 850 & & (3) \\
\hline & SM, UC & 1. 5671 (CUZ) & & CU, HU, \\
\hline 316. & Micrandra sp. 1 (1049WFR) & SM, UC & 348. & Inga \\
\hline & 1800 & 332. Inga acrocephala Steud. & & \\
\hline & K. Garcia, et al. 1049 (CUZ, MO, & $1250-1500$ & & et al. 778 (CUZ, F, USM, \\
\hline & USM, WFU) & rfan, et al. 1909 (CUZ, F, USM, & & WFU) \\
\hline 317. & Sapium glandulosum (L.) Morong & WFU) & & AM, HU, LO, PA \\
\hline & $1500-1750 \mathrm{~m}$ & LO, MD, PA, SM, TU & 349. & Inga nob \\
\hline & K. Garcia, et al. 1080 (CUZ, F, USM, & 333. Inga alba (Sw.) W & & $1250-17$ \\
\hline & & $800-1500$ & & arfan, et al. 1601 (CUZ, F, USM, \\
\hline & AM, CA, CU, HU, JU, LO, MD, PA, & W. Farfan, et al. 1405 (CUZ, F, USM) & & WFU) \\
\hline & SM, TU, UC & AM, CU, JU, LO, MD, PA, SM, UC & & AM, CA, CU, JU, LO, MD, PA, SM, \\
\hline 318. & Sapium laurifolium (A. Rich.) Griseb. & 334. Inga auristellae Harms & & UC \\
\hline & $1250-1500 \mathrm{~m}$ & $800 \mathrm{~m} .^{*}$ & 350. & Inga pezizifera Benth. \\
\hline & W. Farfan, et al. 3969 (CUZ, F, USM) & W. Farfan, et al. 1442 (CUZ, F, USM) & & $1500 \mathrm{~m} . * \wedge$ \\
\hline & AM, CU, HU, LO, MD, PA, PI, SM, & $\mathrm{AM}, \mathrm{LO}, \mathrm{MD}, \mathrm{PA}, \mathrm{SM}$ & & K. Garcia, et al. 1274 (CUZ, F) \\
\hline
\end{tabular}


Tabla 1. Lista de especies arbóreas y afines en los bosques montanos del sureste peruano.

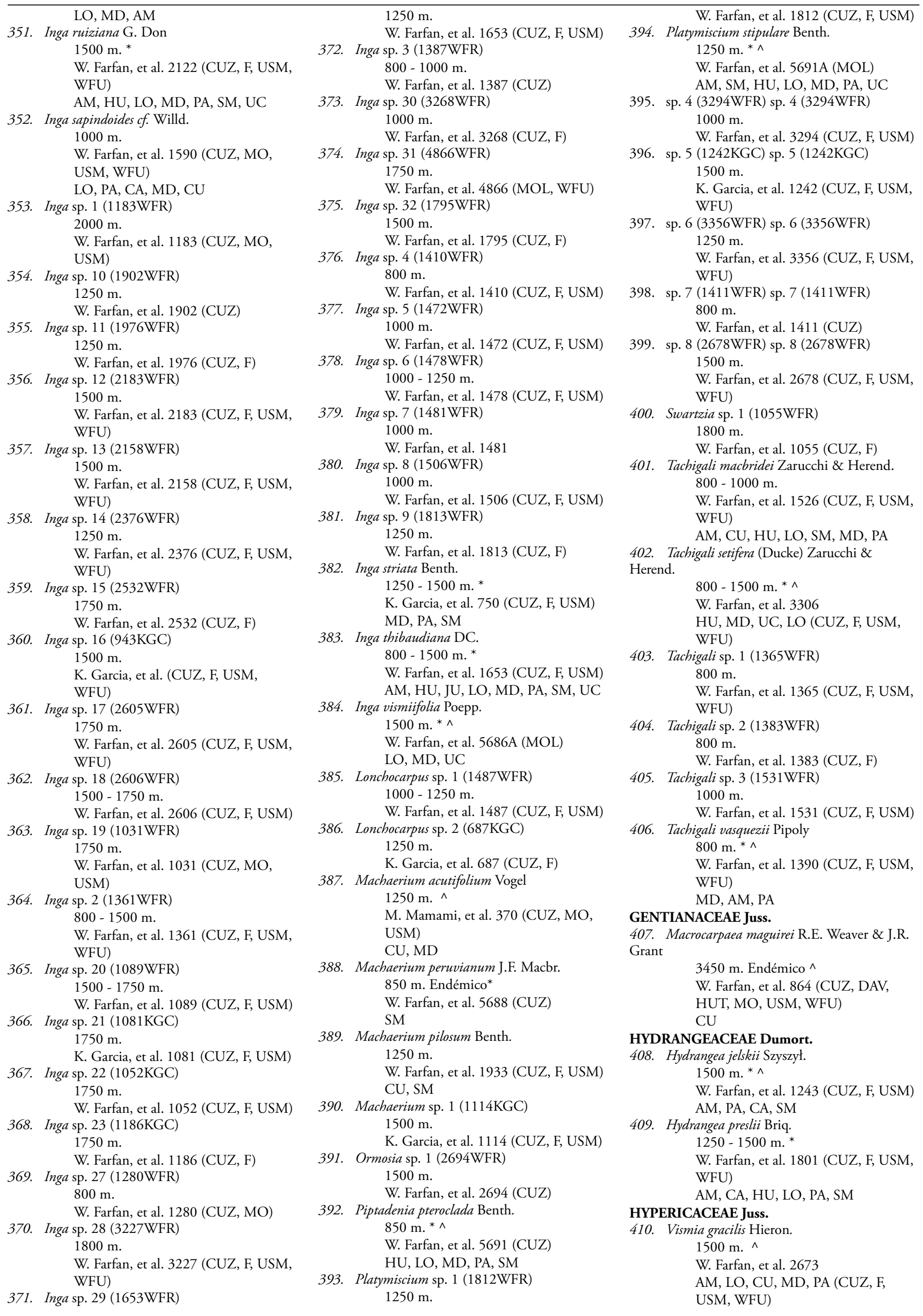


Tabla 1. Lista de especies arbóreas y afines en los bosques montanos del sureste peruano.

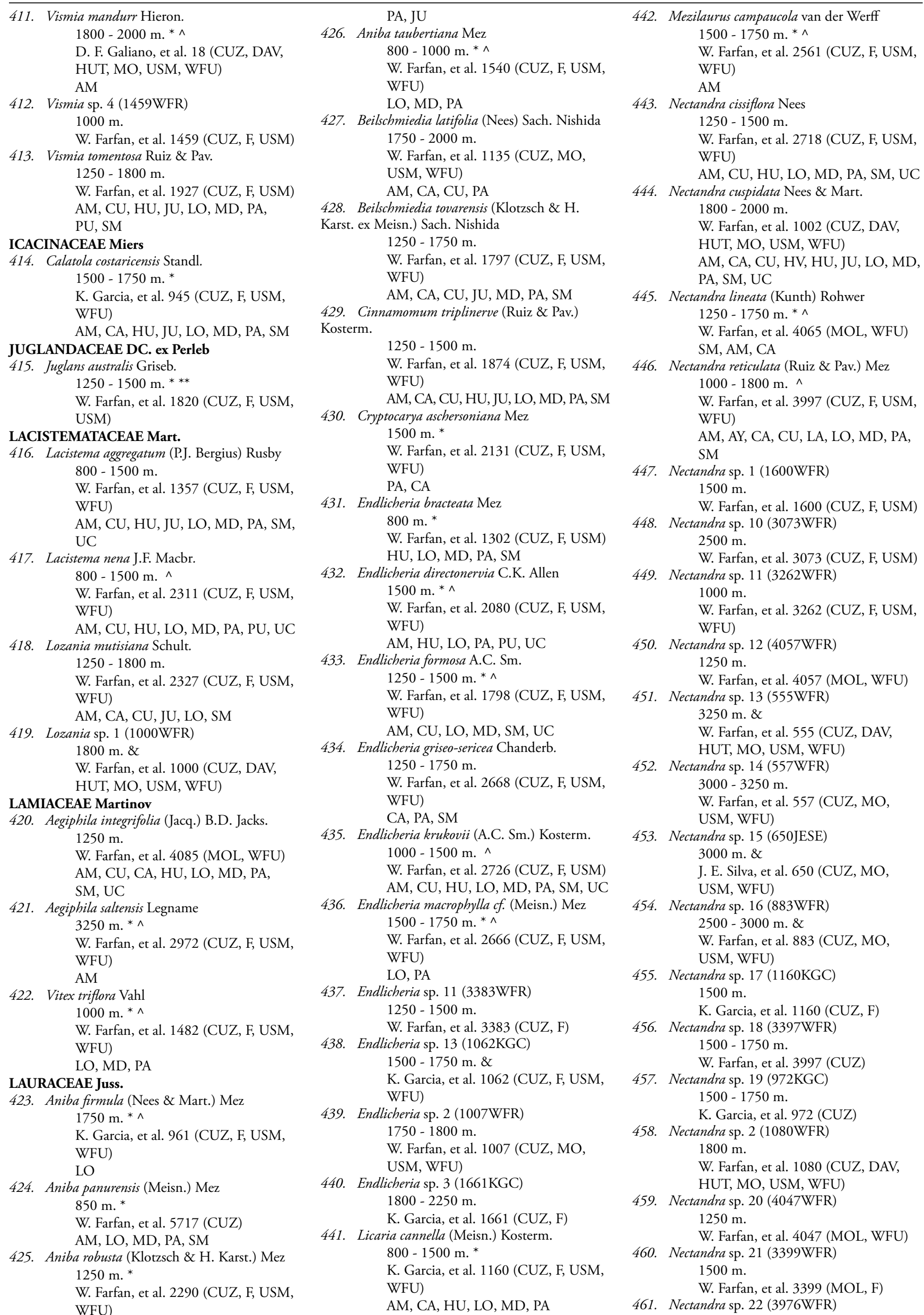


Tabla 1. Lista de especies arbóreas y afines en los bosques montanos del sureste peruano.

\begin{tabular}{|c|c|c|c|c|c|}
\hline & $\begin{array}{l}1500 \mathrm{~m} \text {. } \\
\text { W. Farfan, et al. } 3976 \text { (CUZ, F, USM, } \\
\text { WFU) }\end{array}$ & & $\begin{array}{l}1500 \text { m. }^{* \wedge} \\
\text { W. Farfan, et al. } 1586 \text { (CUZ, F, USM, } \\
\text { WFU) }\end{array}$ & 499. & $\begin{array}{l}\text { Ocotea sp. } 23 \text { (3287WFR) } \\
1000-1250 \mathrm{~m} . \\
\text { W. Farfan, et al. } 3287 \text { (CUZ, F) }\end{array}$ \\
\hline 462. & Nectandra sp. 23 (3384WFR) & & LO, PA, UC, SM & 500. & Ocotea sp. 26 (1626WFR) \\
\hline & $\begin{array}{l}1250 \mathrm{~m} \text {. } \\
\text { W. Farfan, et al. } 3384 \text { (MOL, F, USM) }\end{array}$ & 481. & $\begin{array}{l}\text { Ocotea longifolia Kunth } \\
1250-1500 \mathrm{~m} .\end{array}$ & & $\begin{array}{l}2000 \mathrm{~m} . \\
\text { W. Farfan, et al. } 1626 \text { (CUZ, F, USM) }\end{array}$ \\
\hline 463. & Nectandra sp. 3 (3359WFR) & & W. Farfan, et al. 1819 (CUZ, F, USM, & 501. & Ocotea sp. 28 (1079WFR) \\
\hline & $1000 \mathrm{~m}$ & & WFU) & & $1500-1750 \mathrm{~m}$ \\
\hline 464. & $\begin{array}{l}\text { W. Farfan, et al. } 3359 \text { (MOL, F, USM) } \\
\text { Nectandra sp. } 4 \text { (626KGC) }\end{array}$ & & $\begin{array}{l}\text { AM, CA, CU, HU, LO, MD, PA, } \\
\text { SM, UC }\end{array}$ & & $\begin{array}{l}\text { W. Farfan, et al. } 1079 \text { (CUZ, MO, } \\
\text { USM, WFU) }\end{array}$ \\
\hline & $1000-1500 \mathrm{~m}$ & 482. & Ocotea oblonga (Meisn.) Mez & 502. & Ocotea sp. 4 (1053WFR) \\
\hline & 626 (CUZ, F) & & & & \\
\hline 465. & Nectandra sp & & an, et al. 5713 (CUZ) & & fan, et al. 1053 (CUZ, MO, \\
\hline & $\begin{array}{l}\text { m. } \\
\text { al. } 4008 \text { (MOL, WFU) }\end{array}$ & 483. & $\begin{array}{r}\mathrm{AM}, \\
\text { Ocotea obo }\end{array}$ & 503. & US \\
\hline 466. & Nectandra & & 150 & & 2000 \\
\hline & $\begin{array}{l}1500 \mathrm{~m} \text {. } \\
\text { W. Farfan, et al. } 1696 \text { (CUZ, F, USM) }\end{array}$ & & $\begin{array}{l}\text { W. Farfan, et al. } 2166 \text { (CUZ, F, USM, } \\
\text { WFU) }\end{array}$ & & $\begin{array}{l}\text { W. Farfan, et al. } 1171 \text { (CUZ, MO, } \\
\text { USM, WFU) }\end{array}$ \\
\hline 467. & Nectandra sp. 6 & & $\mathrm{U}, \mathrm{PA}$ & 504. & $4 \mathrm{KGC)}$ \\
\hline & 125 & 484. & (Rich.) Nees & & \\
\hline & n, et al. 1840 (CUZ, F, USM) & & & & et al. 1674 (CUZ, MO, \\
\hline 468. & Nectandra s & & 2205 (CUZ, F, USM) & & \\
\hline & & & $\mathrm{AM}$, & 505. & Ocotea s \\
\hline & CUZ, F) & 485. & Ocotea sp. & & \\
\hline 469. & $\begin{array}{l}\text { Nectandra sp. } 7 \text { (2376AWFR) } \\
1250 \mathrm{~m} .\end{array}$ & & & & W. Farfan, et al. 1045 (CUZ, MO, \\
\hline & n, et al. 2376A (CUZ, F, & & $\begin{array}{l}\text { t al. } 917 \text { (CUZ, MO, } \\
\text { J) }\end{array}$ & 506. & Ocotea sp \\
\hline & & 486. & 3KGC) & & \\
\hline 470. & Nectandra & & & & et al. 576 (CUZ, MO, \\
\hline & & & et al. 1193 (CUZ, F, USM, & & \\
\hline & 404 (CUZ, MO, & & & 507. & Ocotea sp \\
\hline & & 487. & 1 (1654WFR) & & \\
\hline 471. & Nectandra sp. 8 (1643WFR) & & & & W. \\
\hline & & & $\mathrm{Z}, \mathrm{F})$ & 508. & Persea ar \\
\hline & n, et al. 1643 (CUZ, F, USM, & 488. & Ocoteas & & \\
\hline & WFU) & & & & 879 (CUZ, F, USM, \\
\hline 472. & Nectandra sp. 9 (1635WFR) & & fan, et al. 1322 (CUZ, F, USM, & & \\
\hline & & & & & \\
\hline & W. Farfan, et al. 1635 (CUZ, F, USM, & 489. & Ocotea sp & 509. & Persea $b$ \\
\hline 473. & Ocotea aciphylla (Nees \& Mart.) Mez & & 1658 (CUZ, F USM. & & (CUZ, MO, \\
\hline & & & & & \\
\hline & al. 1348 (CUZ, F, USM, & 490. & 4(1884WFR) & & PI \\
\hline & WFU) & & & 510. & Persea caerulea (Ruiz \& Pav.) Mez \\
\hline & A, HU, JU, LO, MD, PA, PU, & & n, et al. 1884 (CUZ, F) & & \\
\hline 474. & $\begin{array}{c}\text { SM } \\
\text { Ocatea hafo }\end{array}$ & 491. & Ocotea & & \\
\hline & $\begin{array}{l}\text { Ocotea bofo } \\
800-\end{array}$ & & & 511 & AN \\
\hline & 3307 (USM) & & & S11. & \\
\hline & , PA, SM & 492. & Ocotea sp. & & et al. 152 (CUZ, MO, \\
\hline 475. & Ocotea ce & & & & \\
\hline & & & arfan, et al. 1308 (CUZ, F, USM, & & \\
\hline & an, et al. 2283 (CUZ, F, USM, & & & 512. & Persea fer \\
\hline & & 493. & Ocotea sp. 18 (1870WFR) & & \\
\hline & $\begin{array}{l}\text { AM, CA, CU, HU, JU, LO, MD, PA, } \\
\text { PU, SM, UC }\end{array}$ & & $\begin{array}{l}12 \\
\text { W. }\end{array}$ & & t al. 811 (CUZ, MO, \\
\hline 476. & (Meisn.) Mez & 494. & Ocotea s & & PI, LA \\
\hline & & & & 513. & Persea $n$ \\
\hline & 5710 (CUZ) & & fan, et al. 2223 (CUZ, F, USM, & & $90 \mathrm{~m} .{ }^{*}$ \\
\hline & & & & & \\
\hline 477. & Ocotea & 495. & Ocotea & & \\
\hline & & & & & \\
\hline & $\begin{array}{l}\text { et al. } 884 \text { (CUZ, MO, } \\
\text { U) }\end{array}$ & & $\begin{array}{l}\text { W. Farfan, et al. } 2119 \text { (CUZ, F, USM, } \\
\text { WFU) }\end{array}$ & 514. & $\begin{array}{r}\text { Persea nudigemn } \\
1250-180\end{array}$ \\
\hline & $\mathrm{CL}$ & 496. & Ocotea sp. 20 & & tal. 1068 (CUZ, MO, \\
\hline 478. & Ocotea insularis (Meisn.) Mez & & & & \\
\hline & & & a, et al. 682 (CUZ, F, USM, & & \\
\hline & arfan, et al. 1335 (CUZ, MO, & & WFU) & 515. & Persea peruviana Nees \\
\hline & & 497. & Ocotea sp. 21 (988KGC) & & \\
\hline & & & & & et al. 1957 (CUZ, F, USM \\
\hline 479. & Ocotea javitensis (Kunth) Pittier & & K. Garcia, et al. 988 (CUZ, F) & & \\
\hline & $850 \mathrm{~m}$. & 498. & Ocotea sp. 22 (2295AWFR) & & $\mathrm{AM}, \mathrm{LO}, \mathrm{JU}$, \\
\hline & W. Farfan, et al. 5724 (CUZ) & & & 516. & Persea sp. 1 (868WFR) \\
\hline & & & Farfan, et al. 2295A (CUZ, F, & & $2500-2750$ m. \& \\
\hline 480. & Ocotea leucoxylon (Sw.) & & USM, WFU) & & W. Farfan, et al. 868 (CUZ, MO, \\
\hline
\end{tabular}


Tabla 1. Lista de especies arbóreas y afines en los bosques montanos del sureste peruano.

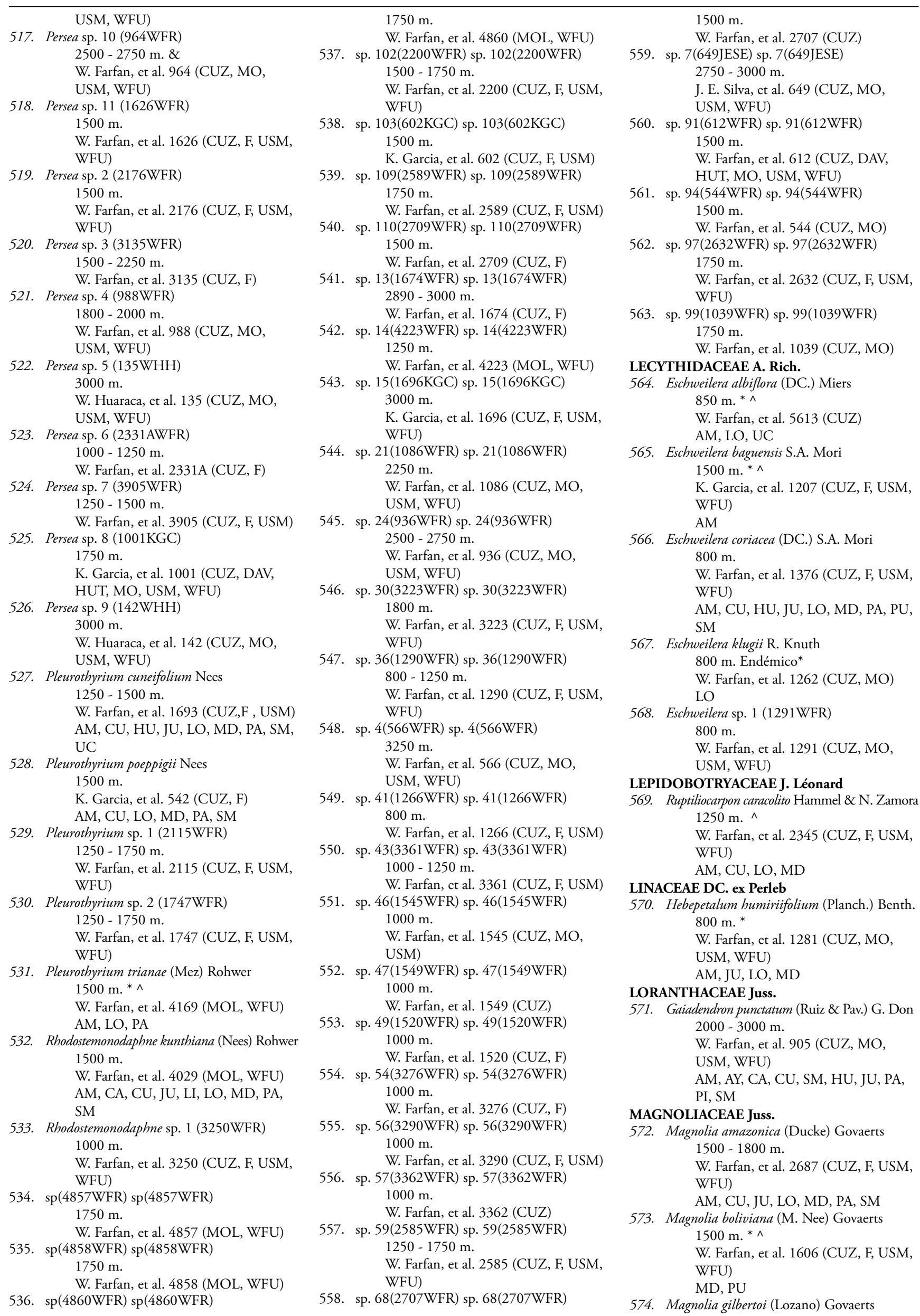


Tabla 1. Lista de especies arbóreas y afines en los bosques montanos del sureste peruano.

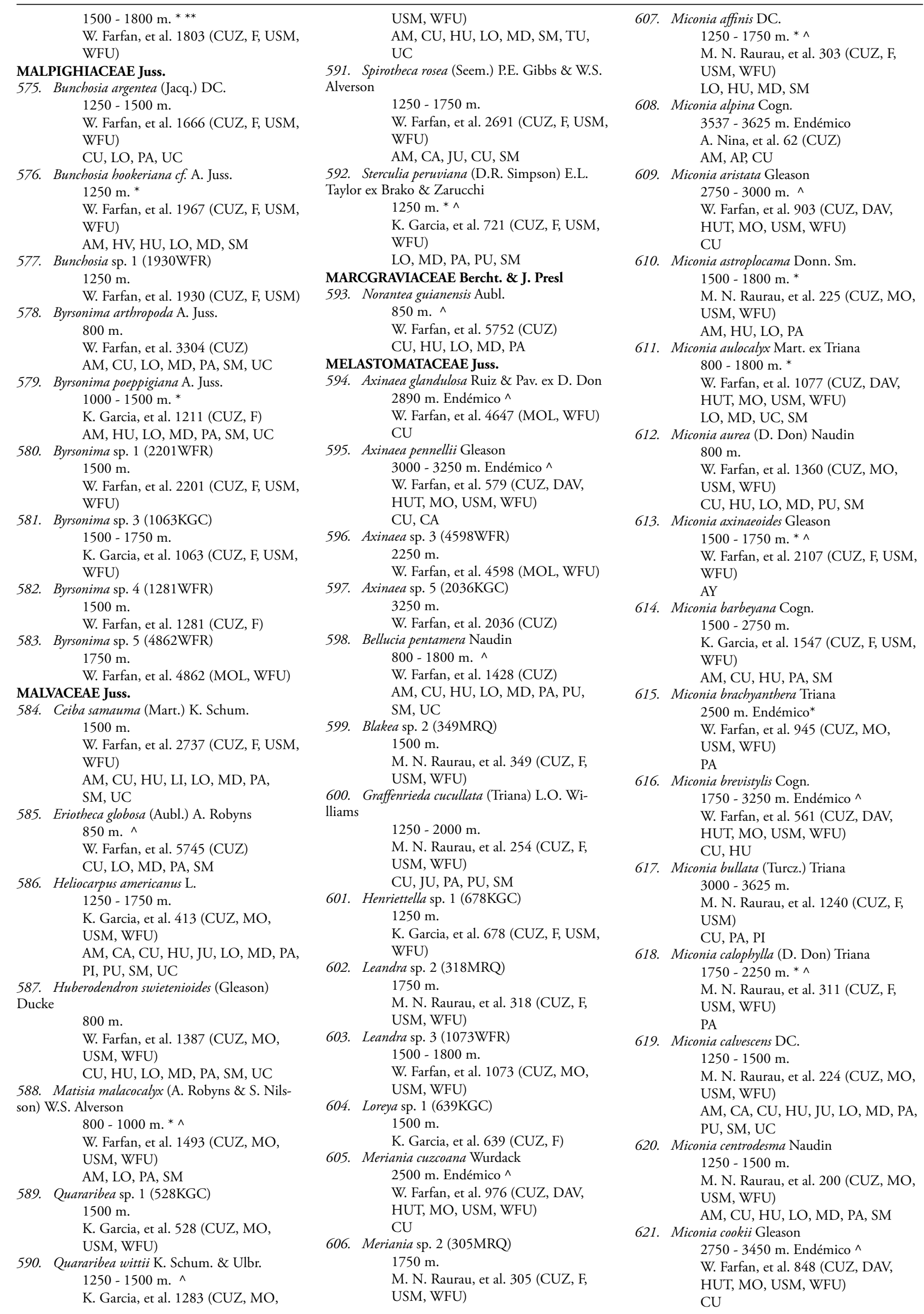


Tabla 1. Lista de especies arbóreas y afines en los bosques montanos del sureste peruano.

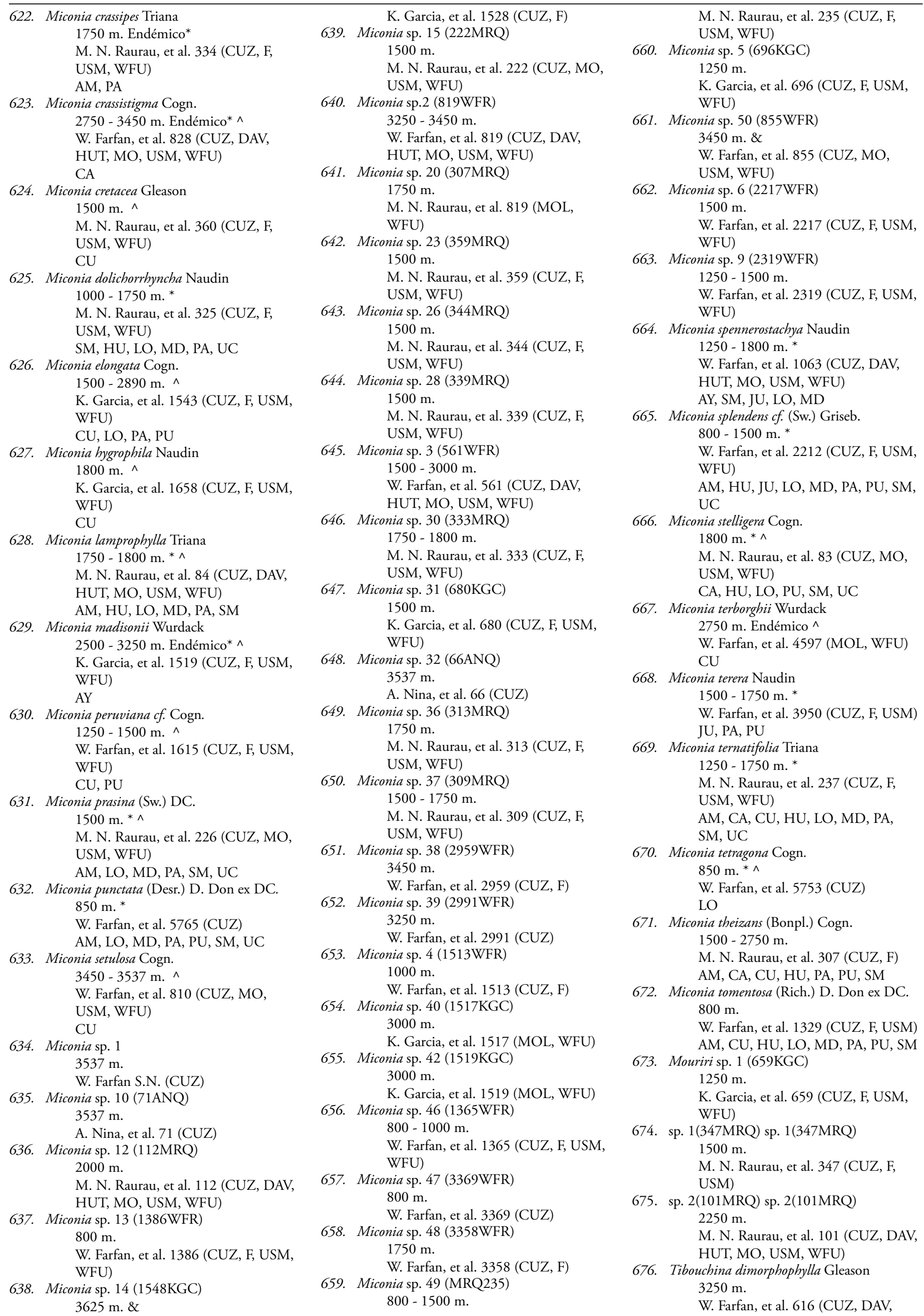


Tabla 1. Lista de especies arbóreas y afines en los bosques montanos del sureste peruano.

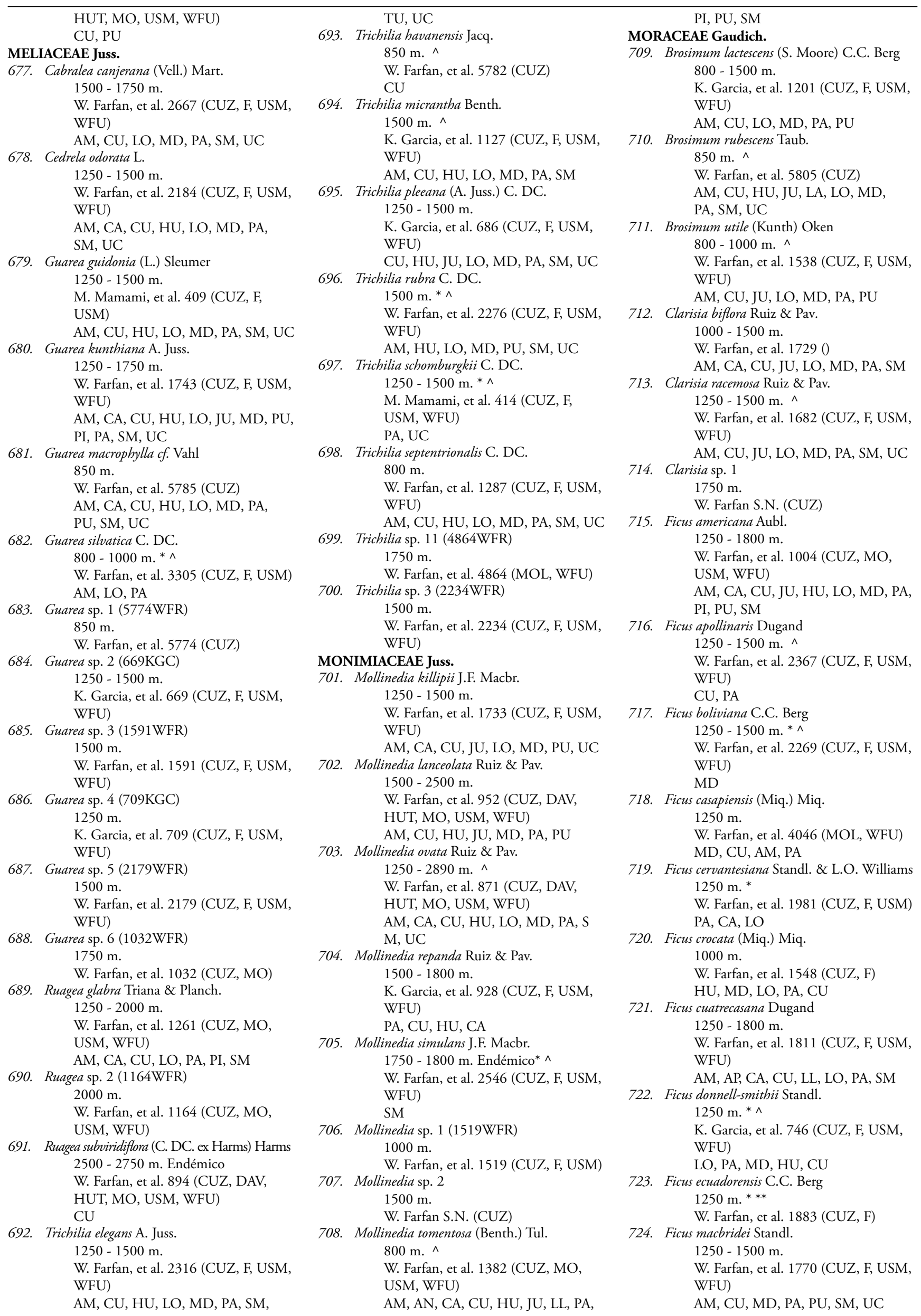


Tabla 1. Lista de especies arbóreas y afines en los bosques montanos del sureste peruano.

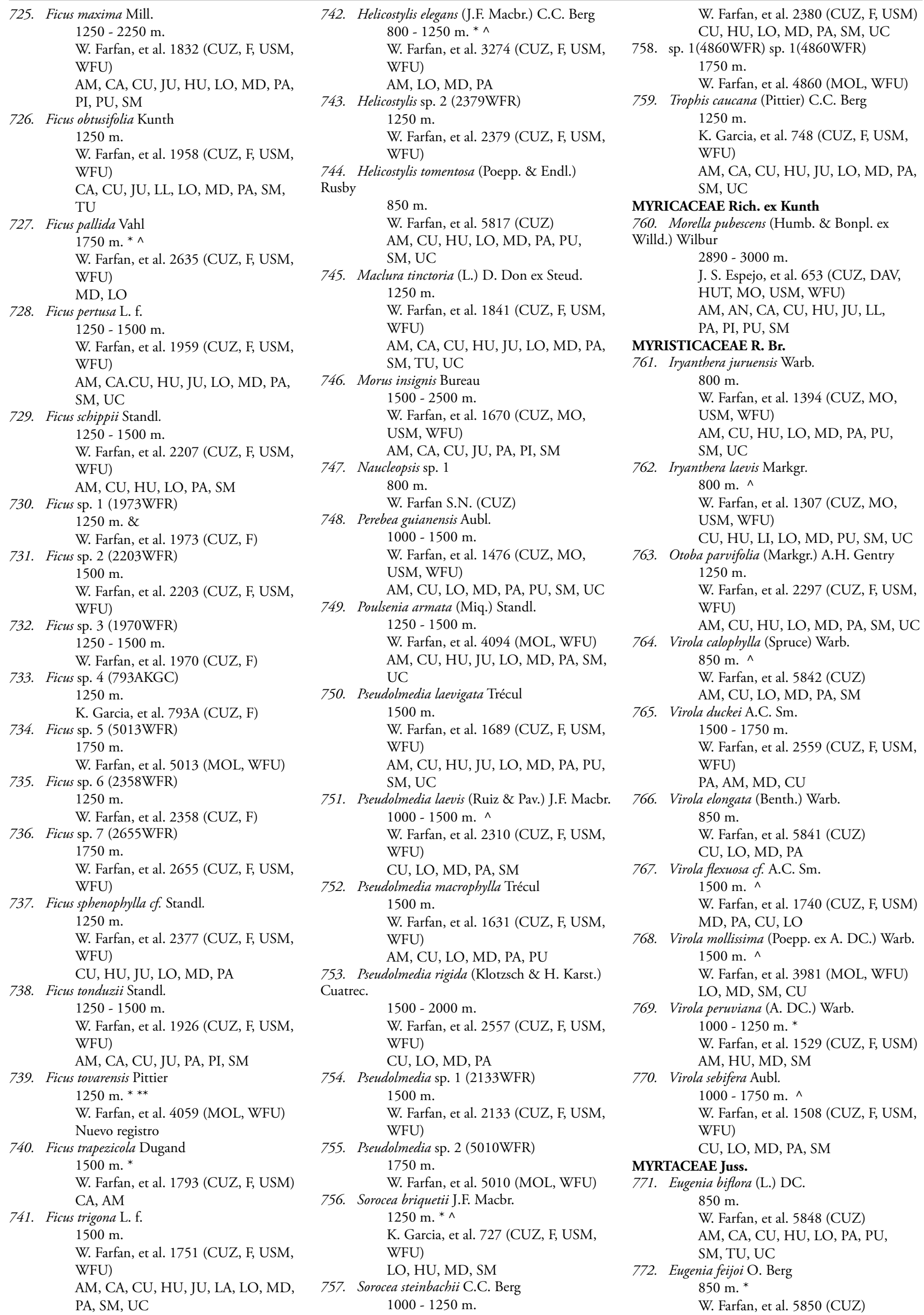


Tabla 1. Lista de especies arbóreas y afines en los bosques montanos del sureste peruano.

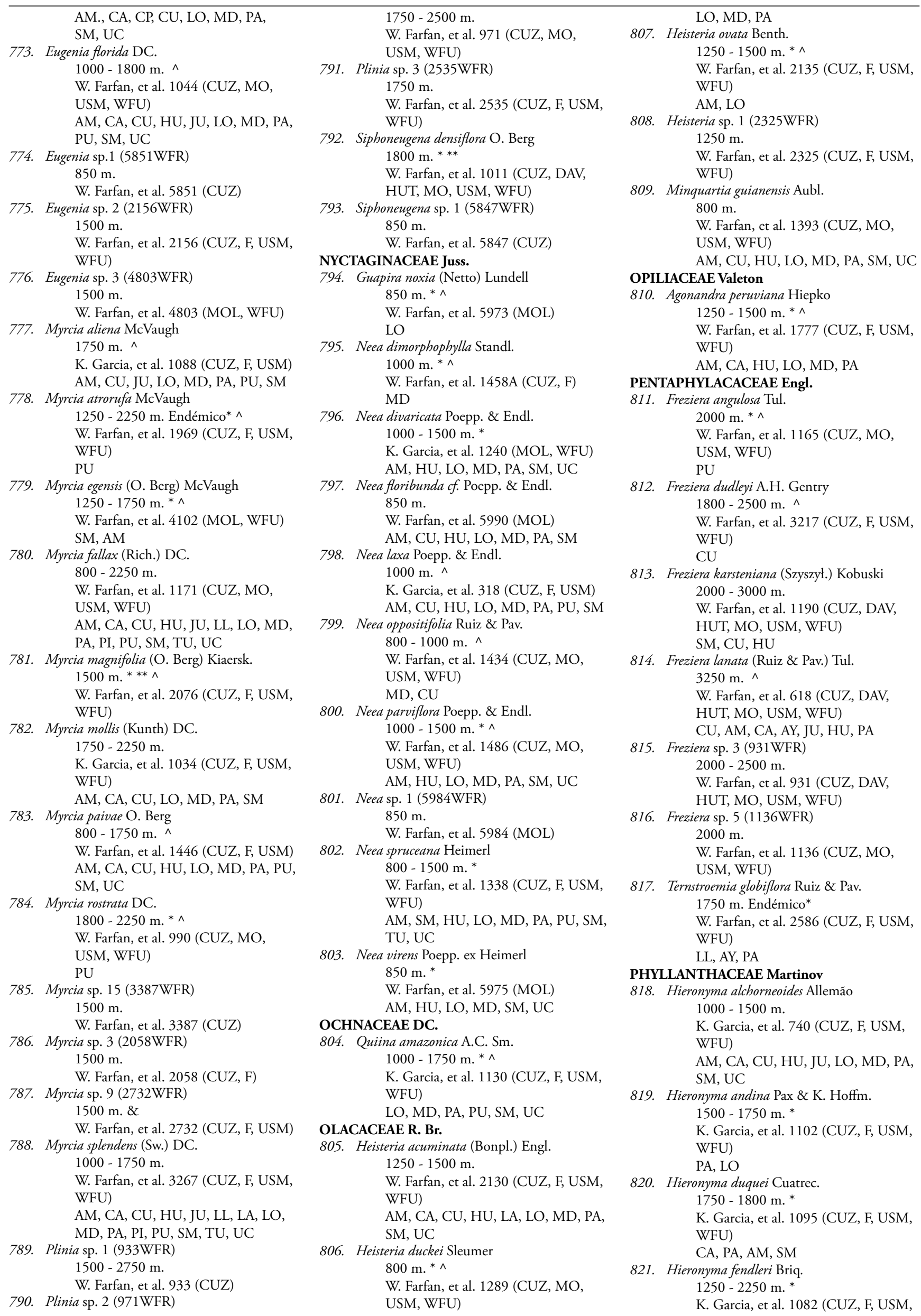


Tabla 1. Lista de especies arbóreas y afines en los bosques montanos del sureste peruano.

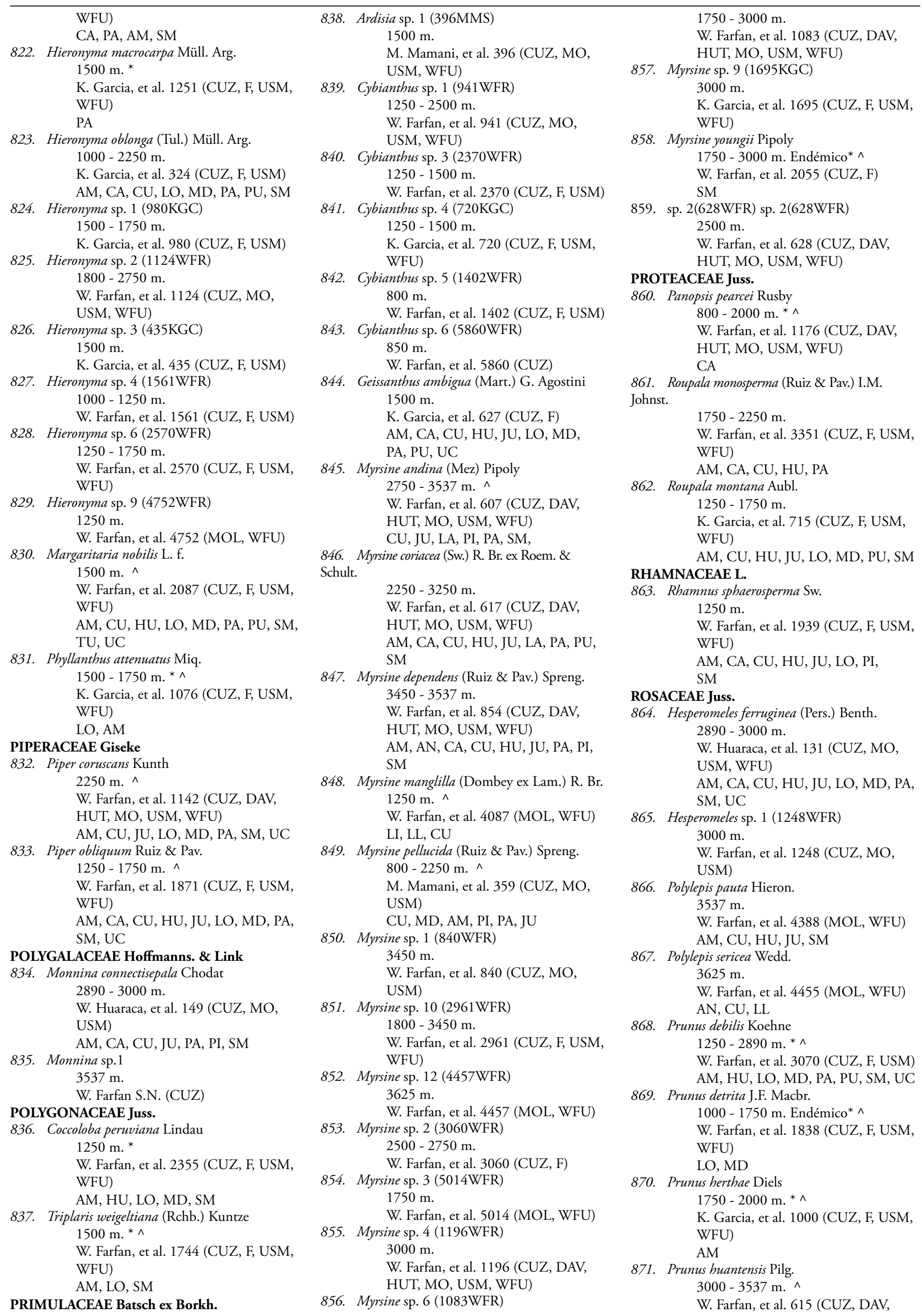


Tabla 1. Lista de especies arbóreas y afines en los bosques montanos del sureste peruano.

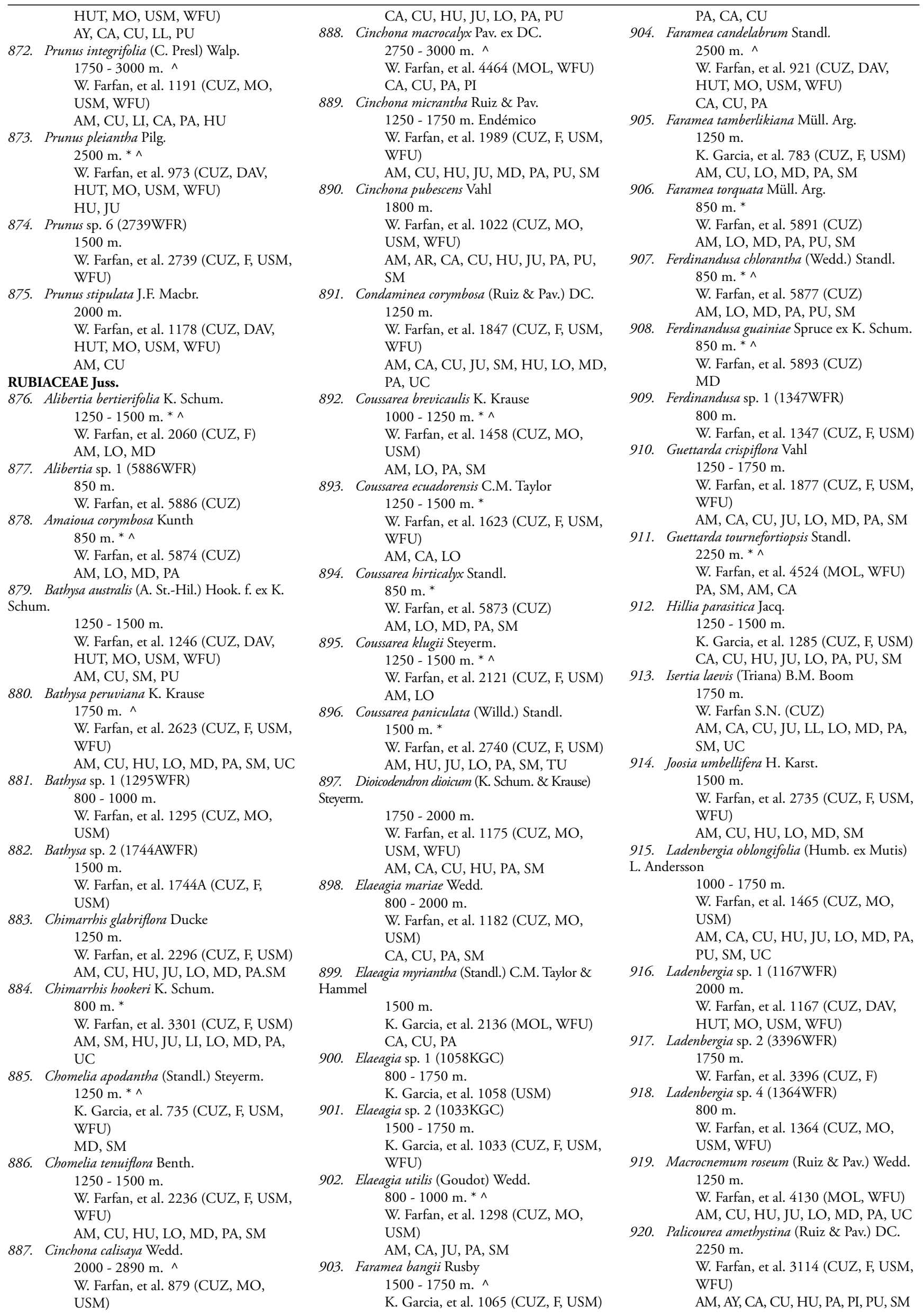


Tabla 1. Lista de especies arbóreas y afines en los bosques montanos del sureste peruano.

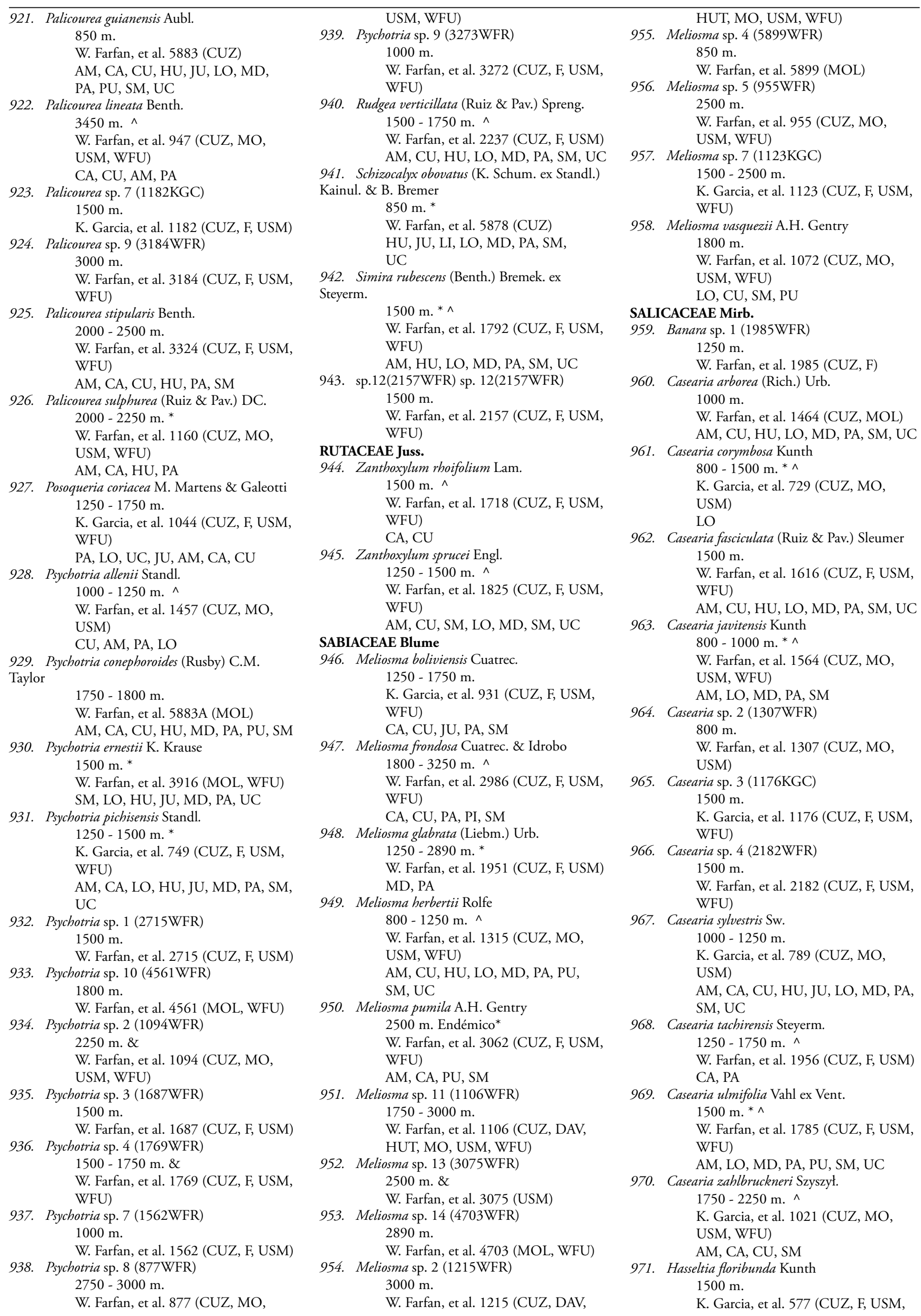


Tabla 1. Lista de especies arbóreas y afines en los bosques montanos del sureste peruano.

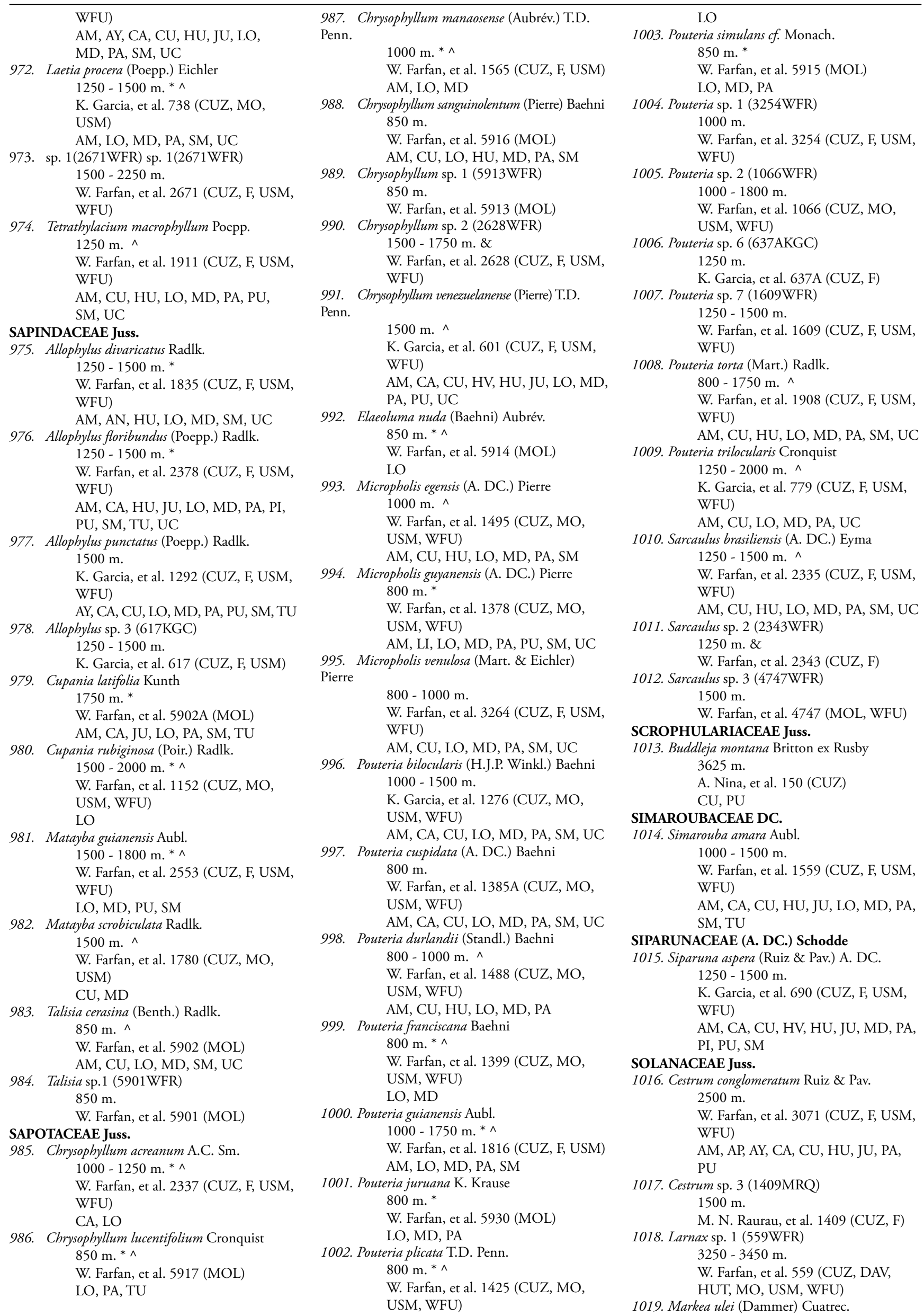


Tabla 1. Lista de especies arbóreas y afines en los bosques montanos del sureste peruano.

\begin{tabular}{|c|c|c|}
\hline $1250 \mathrm{~m} . \wedge$ & W. Farfan, et al. 3404 (CUZ, F, USM) & AM, CU, PA \\
\hline K. Garcia, et al. 724 (CUZ, F, USM, & 1038. sp. 41(3405WFR) sp. 41(3405WFR) & 1055. Symplocos sp. 4 (3138WFR) \\
\hline WFU) & $800 \mathrm{~m}$ & $2000-2250$ m. \& \\
\hline AM, CU, HU, LO, MD, PA, SM, UC & W. Farfan, et al. 3405 (CUZ) & W. Farfan, et al. 3138 (CUZ, F, USM) \\
\hline 1020. Saracha punctata Ruiz \& Pav. & 1039. sp. 42(3406WFR) sp. 42(3406WFR) & 1056. Symplocos sp. 5 (4165WFR) \\
\hline $3250-3625 \mathrm{~m}$ & $800 \mathrm{~m}$ & \\
\hline W. Farfan, et al. 849 (CUZ, MO, USM) & W. Farfan, et al. 3406 (CUZ) & W. Farfan, et al. 4165 (MOL, WFU) \\
\hline AN, CA, CU, HU, JU, PA, PI, PU, SM & 1040. sp. 43(3407WFR) sp. 43(3407WFR) & 1057. Symplocos spruceana Gürke \\
\hline 1021. Sessea dependens Ruiz \& Pav. & $800 \mathrm{~m}$ & $2000-2890$ \\
\hline $3537-3625 \mathrm{~m}$ & W. Farfan, et al. 3407 (CUZ) & W. Farfan, et al. 3107 (CUZ, F, USM, \\
\hline et al. 21 (CUZ) & 1041. sp. $47(34$ & WFU) \\
\hline $\mathrm{CU}$ & $1500 \mathrm{~m}$ & CU, SM \\
\hline 1022. Solanum aphyodendron S. Knapp & W. Farfan, et al. 3409 (CUZ, F) & THEACEAE Mirb. \\
\hline $1500 \mathrm{~m}$ & 1042. sp. 6(3312WFR) sp. 6(3312WFR) & 1058. Gordonia fruticosa (Schrad.) H. Keng \\
\hline $\begin{array}{l}\text { W. Farfan, et al. } 1778 \text { (CUZ, F, USM, } \\
\text { WFU) }\end{array}$ & $\begin{array}{l}800 \mathrm{~m} . \\
\text { W. Farfan, et al. } 3312 \text { (CUZ, F, USM) }\end{array}$ & $\begin{array}{l}800-2750 \mathrm{~m} . \\
\text { W. Farfan, et al. } 880 \text { (CUZ, MO, }\end{array}$ \\
\hline P, AY, CA, CU, HU, LI, LO, & 1043. sp. $8(326$ & USN \\
\hline $\mathrm{PA}, \mathrm{P}$ & 100 & HU, JU, LO, PA \\
\hline 1023. Solanum grandiflorum Ruiz \& Pav. & fan, et al. 3266 (CUZ, F, USM) & 1059. Gordonia pubescens L'Hér. \\
\hline $1250 \mathrm{~m}$ & STAPHYLEACEAE Martinov & $1000 \mathrm{~m} . * * *$ \\
\hline $\begin{array}{l}\text { W. Farfan, et al. } 1912 \text { (CUZ, F, USM, } \\
\text { WFU) }\end{array}$ & $\begin{array}{r}\text { 1044. Staphylea occ } \\
1250-\end{array}$ & $\begin{array}{l}\text { W. Farfan, et al. } 1453 \text { (CUZ, MO, } \\
\text { USM, WFU) }\end{array}$ \\
\hline IU, JU, LO, MD, PA, SM, UC & l. 1150 (CUZ, DAV, & 1060. Ternstroemia brachypoda (Wawra) Kobuski \\
\hline 1024. Solanum lanceolatu & U) & $2750 \mathrm{~m} . \wedge$ \\
\hline 150 & AM, CA, CU, HU, LO, MD, PA, & K. Garcia, et al. 1544 (CUZ, F, USM, \\
\hline , et al. 603 (CUZ, F, USM, & & \\
\hline 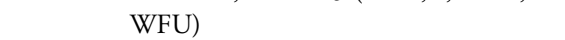 & STYRACACE & $\mathrm{CU}$ \\
\hline $\mathrm{HU}$ & 1045. Styrax fov & ULMACEAI \\
\hline 1025. Solanum maturecalvans Bitter & & dentula Kuhlm. \\
\hline 2250 & arfan, et al. 1188 (CUZ, MO, & \\
\hline 575 (CUZ, DAV, & & arfan, et al. 2271 (CUZ, \\
\hline HU1 & & \\
\hline AY, CA, CU, JU, LL, & 1046. Styrax pentlandianus J. Rémy & $\mathrm{O}, \mathrm{MD}, \mathrm{PA}, \mathrm{SM}, \mathrm{UC}$ \\
\hline $\mathrm{U}, \mathrm{SM}$ & & 1062. Ampelo \\
\hline 1026. Solanum & K. Garcia, et al. 1682 (CUZ, F, USM, & $1500 \mathrm{~m}$ \\
\hline 225 & & rfan, et al. 2253 (CUZ, F, USM, \\
\hline 881 (CUZ, DAV, & & J) \\
\hline $\mathrm{HU}$ & 1047. Styrax sie & $\mathrm{HU}, \mathrm{LO}, \mathrm{MD}, \mathrm{PA}, \mathrm{SM}$ \\
\hline , AY, CA, CU, HU, JU, LL, & & URTICACEAE \\
\hline LA, I & W. Farfan, et al. 3251 (CUZ, F, USM, & 1063. Cecropic \\
\hline 1027. Solanum $\mathrm{sp}$ & & \\
\hline $1500 \mathrm{~m}$ & CA & an, et al. 1021 (CUZ, MO, \\
\hline al. 1290 (CUZ, F, USM, & SYMPLOCAC & \\
\hline WFU & 1048. Symploc & I PA SM \\
\hline 1028. sp. $14(733$ & 1500 & 1064. Cecropia membra \\
\hline 125 & 4165 (MOL, WFU) & \\
\hline a, et al. 733 (CUZ) & & al. 3993 (CUZ, F, USM, \\
\hline 1029. sp. $15(33$ & 1049. Symploco & \\
\hline $1250 \mathrm{~m}$ & & AM, HU, LI, LO, MD, PA, SM, TU \\
\hline an, et al. 3390 (CUZ, F) & al. 609 (CUZ, MO, & 1065. Cecropia polystachy \\
\hline 1030. sp. $19(1$ & & \\
\hline $1750 \mathrm{~m}$ & $\mathrm{CU}$ & W \\
\hline al. 1042 (CUZ, F, USM, & 1050. Symplocos fuliginosa B. Ståhl & $\mathrm{CU}, \mathrm{HU}$ \\
\hline WFL & & 1066. Cecropia \\
\hline 1031. sp. 22(1029KGC) sp. 22(1029KGC) & W. Farfan, et al. 2111 (CUZ, F, USM, & $1500 \mathrm{~m}$ \\
\hline & & \\
\hline K. G & & 1067. Cecropia \\
\hline 1032. sp. $28(2753)$ & 1051. Symploco & \\
\hline & & $(\mathrm{CUZ}, \mathrm{F})$ \\
\hline W. Fa & t al. 1118 (CUZ, MO, & 1068. Cecropia \\
\hline 1033. sp. $37(3401$ & & \\
\hline & $\mathrm{CA}$ & M. N. Raurau, et al. 555 (CUZ, F, \\
\hline al. 3401 (CUZ, F, USM & 1052. Symplocos psilo & \\
\hline & & 1069. Cecropia \\
\hline 1034. sp. 38(3402WFR) sp. 38(3402WFR) & W. Farfan, et al. 815 (CUZ, DAV, & $1750 \mathrm{~m}$ \\
\hline & & W. Farfan, et al. 2652 (CUZ, F, USM, \\
\hline W. Fa & & \\
\hline 1035. sp. 39(3403WFR) sp. 39(3403WFR) & 1053. Symplocos quitensis Brand & 1070. Cecropia sp. 3 (1855WFR) \\
\hline $800 \mathrm{~m}$ & $3250-3$ & $1250 \mathrm{~m}$ \\
\hline $3(\mathrm{CUZ}, \mathrm{F})$ & 1 (CUZ, MO) & fan, et al. 1855 (CUZ, F, USM) \\
\hline 1036. sp. 4(1880WFR) sp. 4(1880WFR) & & 1071. Cecropia sp. 4 (1855AWFR) \\
\hline $1250 \mathrm{~m}$ & 1054. Symplocos reflexa A. DC. & $1250 \mathrm{~m}$ \\
\hline W. Farfan, et al. 1880 (CUZ, F, USM) & $2750-3000 \mathrm{~m}$ & W. Farfan, et al. 1855A (CUZ, F) \\
\hline 1037. sp. 40(3404WFR) sp. 40(3404WFR) & W. Farfan, et al. 1194 (CUZ, DAV, & 1072. Cecropia sp. 5 (160AVHQ) \\
\hline $800 \mathrm{~m}$ & HUT, MO, USM, WFU) & $1000 \mathrm{~m}$ \\
\hline
\end{tabular}


Tabla 1. Lista de especies arbóreas y afines en los bosques montanos del sureste peruano.

\begin{tabular}{|c|c|c|}
\hline V. Huaman, et al. 160A (CUZ, F, & 1086. Pourouma herrerensis C.C. Berg & VITACEAE Juss. \\
\hline USM) & $1250-1500 \mathrm{~m}$. Endémico*^ & 1099. Cissus trianae Planch. \\
\hline 1073. Cecropia sp. 6 (691KGC) & W. Farfan, et al. 2308 (CUZ, F, USM, & $1750 \mathrm{~m}$ \\
\hline $1250 \mathrm{~m}$ & WFU) & W. Farfan, et al. 1440 (CUZ, F, USM, \\
\hline K. Garcia, et al. 691 (CUZ, F, USM, & LO & WFU) \\
\hline WFU) & 1087. Pourouma minor Benoist & AM, CA, CU, PA, SM \\
\hline 1074. Cecropia sp. 7 (1839WFR) & $800-1500 \mathrm{~m}$ & VOCHYSIACEAE A. St.-Hil. \\
\hline $1250 \mathrm{~m}$ & W. Farfan, et al. 3309 (CUZ, F, USM) & 1100. Qualea paraensis Ducke \\
\hline W. Farfan, et al. 1839 (CUZ, F) & $\mathrm{CU}, \mathrm{HU}, \mathrm{LO}, \mathrm{MD}, \mathrm{PA}, \mathrm{SM}, \mathrm{UC}$ & $850 \mathrm{~m} . \wedge$ \\
\hline 1075. Cecropia sp. 8 (2575WFR) & 1088. Pourouma mollis Trécul & W. Farfan S.N. (CUZ) \\
\hline $1750 \mathrm{~m}$ & $800-1750 \mathrm{~m} .{ }^{*}$ & AM, CU, LO, MD, SM \\
\hline W. Farfan, et al. 2575 (CUZ) & V. Huaman, et al. 68 (CUZ, F, USM) & 1101. Vochysia biloba Ducke \\
\hline 1076. Cecropia sp. 9 (624JESE) & HU, JU, LO, MD, PA, SM, UC & $800-1000 \mathrm{~m}$ \\
\hline $2000-2500$ m. \& & 1089. Pourouma sp. 2 (1413WFR) & W. Farfan, et al. 1532 (CUZ, F, USM) \\
\hline J. E. Silva, et al. 624 (CUZ, MO, & $800 \mathrm{~m}$ & $\mathrm{AM}, \mathrm{CU}, \mathrm{LO}$ \\
\hline USM, WFU) & W. Farfan, et al. 1413 (CUZ, F) & 1102. Vochysia kosnipatae Huamantupa \\
\hline 1077. Coussapoa sp. 1 (1356WFR) & 1090. Pourouma sp. 3 (160VHQ) & $1250 \mathrm{~m}$. Endémico \\
\hline $800 \mathrm{~m}$ & $1000 \mathrm{~m}$ & W. Farfan, et al. 2339 (CUZ, F, USM, \\
\hline W. Farfan, et al. 1356 (CUZ, MO, & V. Huaman, et al. 160 (CUZ, F, USM) & WFU) \\
\hline USM, WFU) & 1091. Pourouma sp. 7 (174VHQ) & CU, PA \\
\hline 1078. Coussapoa sp. 2 (2244WFR) & $800-1000 \mathrm{~m}$ & 1103. Vochysia leguiana J.F. Macbr. \\
\hline $1500 \mathrm{~m}$ & V. Huaman, et al. 174 (CUZ, F, USM, & $1000 \mathrm{~m} . * \wedge$ \\
\hline W. Farfan, et al. 2244 (CUZ, F, USM) & WFU) & W. Farfan, et al. 3298 (CUZ, F, USM, \\
\hline 1079. Coussapoa sp. 3 (713KGC) & 1092. Urera baccifera (L.) Gaudich. ex Wedd. & WFU) \\
\hline $1250 \mathrm{~m}$ & $1500 \mathrm{~m}$ & JU, SM \\
\hline K. Garcia, et al. 713 (CUZ, F, USM, & K. Garcia, et al. 1282 (CUZ, MO, & 1104. Vochysia sp. 1 (1356WFR) \\
\hline WFU) & USM) & $800 \mathrm{~m}$ \\
\hline 1080. Coussapoa villosa Poepp. \& Endl. & AM, CA, CU, HU, JU, LO, MD, PA, & W. Farfan, et al. $1356(\mathrm{CUZ}, \mathrm{F})$ \\
\hline $1500 \mathrm{~m} .^{*}$ & SM, TU, UC & 1105. Vochysia sp. 2 (3255WFR) \\
\hline K. Garcia, et al. 1280 (CUZ, F, USM, & 1093. Urera caracasana (Jacq.) Gaudich. ex Griseb. & $1000 \mathrm{~m} . \&$ \\
\hline WFU) & $1250-2250 \mathrm{~m} . \wedge$ & W. Farfan, et al. 3255 (USM, WFU) \\
\hline AM, HU, JU, LI, LO, MD, PA, SM, UC & W. Farfan, et al. 1657 (CUZ, F, USM, & 1106. Vochysia sp. 3 (3314WFR) \\
\hline 1081. Myriocarpa stipitata Benth. & WFU) & $800 \mathrm{~m}$ \\
\hline $1250 \mathrm{~m}$ & CA, MD, LO, PA, HU, UC, JU, CU, SM & W. Farfan, et al. 3314 (USM) \\
\hline K. Garcia, et al. 489 (CUZ, MO) & 1094. Urera simplex Wedd. & 1107. Vochysia sp. 4 (2743WFR) \\
\hline AM, CA, CU, HU, JU, LO, MD, PA, & $1250 \mathrm{~m}$ & $1500 \mathrm{~m}$ \\
\hline $\mathrm{SM}, \mathrm{UC}$ & K. Garcia, et al. 469 (CUZ, F, USM) & W. Farfan, et al. 2743 (CUZ, F, USM, \\
\hline 1082. Pourouma bicolor Mart. & AM, MD, CU, HU, LO, PA, SM & WFU) \\
\hline $1500-1750 \mathrm{~m} .{ }^{*}$ & 1095. Urera sp. 1 (2048WFR) & 1108. Vochysia sp. 5 (1384WFR) \\
\hline K. Garcia, et al. 1140 (CUZ, F, USM) & $1500 \mathrm{~m}$ & $800 \mathrm{~m}$ \\
\hline AM, LO, MD, HU, PA, SM & W. Farfan, et al. 2048 (CUZ, F) & W. Farfan, et al. 1384 (CUZ, MO, \\
\hline 1083. Pourouma cecropiifolia Mart. & VIOLACEAE Batsch & USM, WFU) \\
\hline $800 \mathrm{~m}$ & 1096. Leonia glycycarpa Ruiz \& Pav. & \\
\hline W. Farfan, et al. 3310 (CUZ, F, USM) & $800-1000 \mathrm{~m} \cdot \wedge$ & \\
\hline AM, CU, LO, MD, PA, PU, SM, UC & W. Farfan, et al. 1501 (CUZ, F, USM) & \\
\hline 1084. Pourouma cuspidata Mildbr. & AM, CU, HU, JU, LO, MD, PA, PU, & \\
\hline $1000-1250 \mathrm{~m} .{ }^{*} \wedge$ & SM, UC & \\
\hline W. Farfan, et al. 2368 (CUZ, F, USM, & 1097. Rinorea apiculata Hekking & \\
\hline WFU) & $1250 \mathrm{~m} \cdot \wedge$ & \\
\hline MD, LO, PA & W. Farfan, et al. 4007 (MOL, WFU) & \\
\hline 1085. Pourouma guianensis Aubl. & AM, HU, CU, LO, MD & \\
\hline $850 \mathrm{~m}$ & 1098. Rinorea sp. 1 (1454WFR) & \\
\hline W. Farfan, et al. 5954 (MOL) & $1000 \mathrm{~m}$ & \\
\hline $\begin{array}{l}\text { AM, CA, CU, HU, LO, MD, PA, } \\
\text { SM, UC }\end{array}$ & W. Farfan, et al. 1454 (CUZ, F, USM) & \\
\hline
\end{tabular}



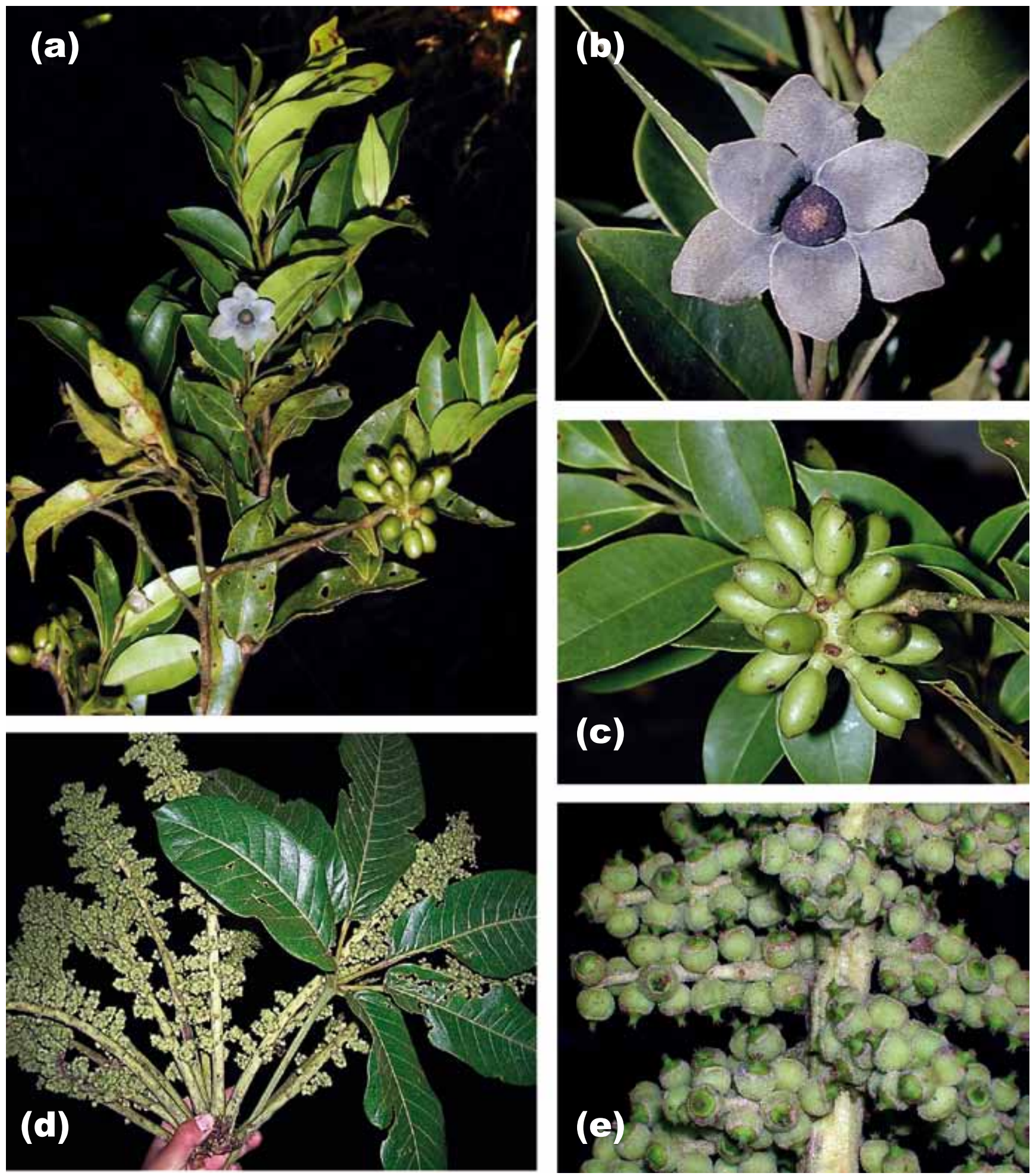

Anexo 1. Guatteria terminalis [W. Farfan, et al. 1112] (a), flor (b), frutos (c). Schefflera sp. 1 (158WHH) sp. Nova [W. Huaraca, et al. 158] (d) frutos (e). Credito de fotos: W. Farfan Rios. 

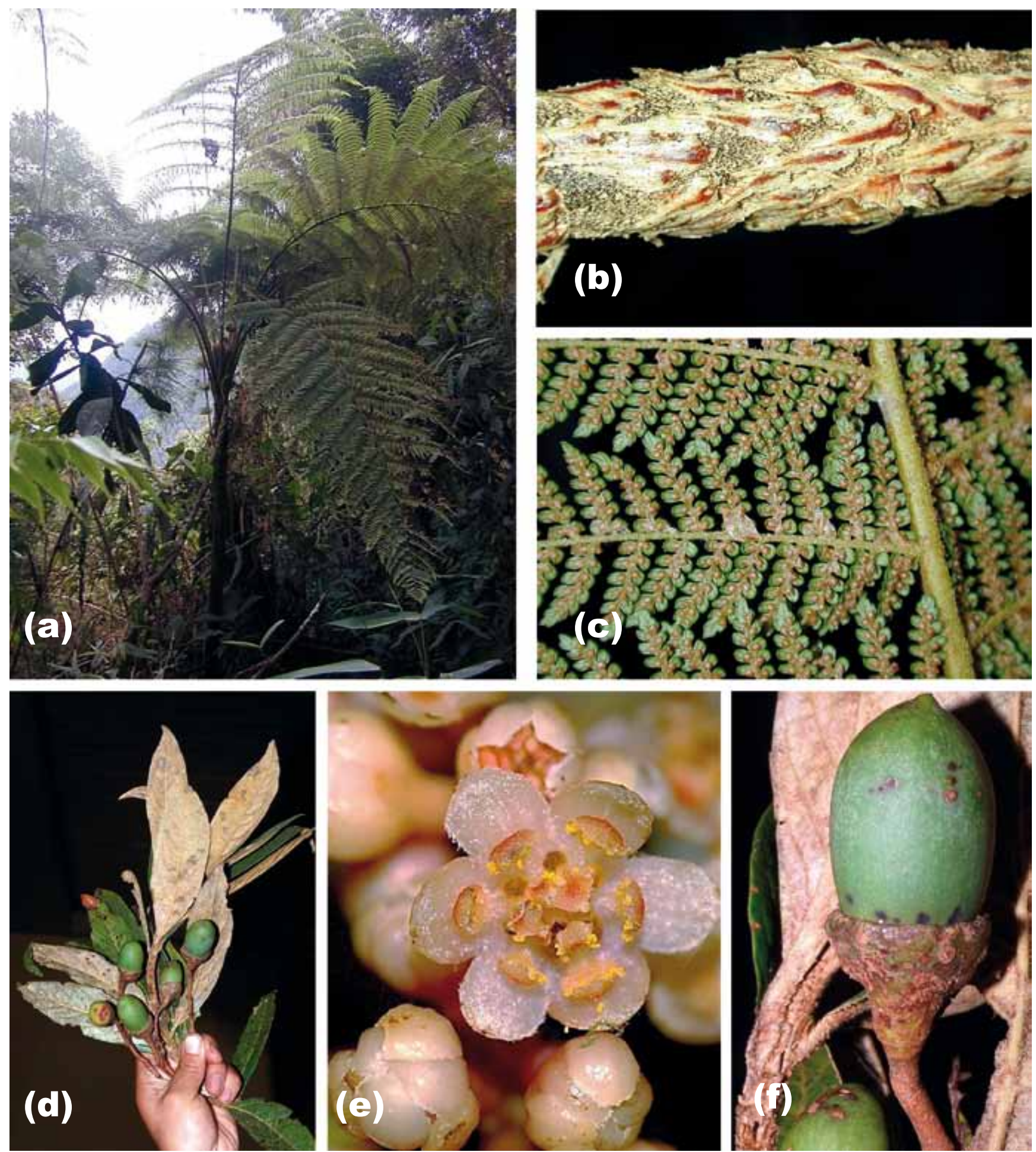

Anexo 2. Cyathea multisegmenta [K. Garcia, et al. 208] (a), escamas (b), soros (c). Ocotea glabriflora [W. Farfan, et al. 884] (d), flor (e), fruto (f). Credito de fotos: W. Farfan Rios. 

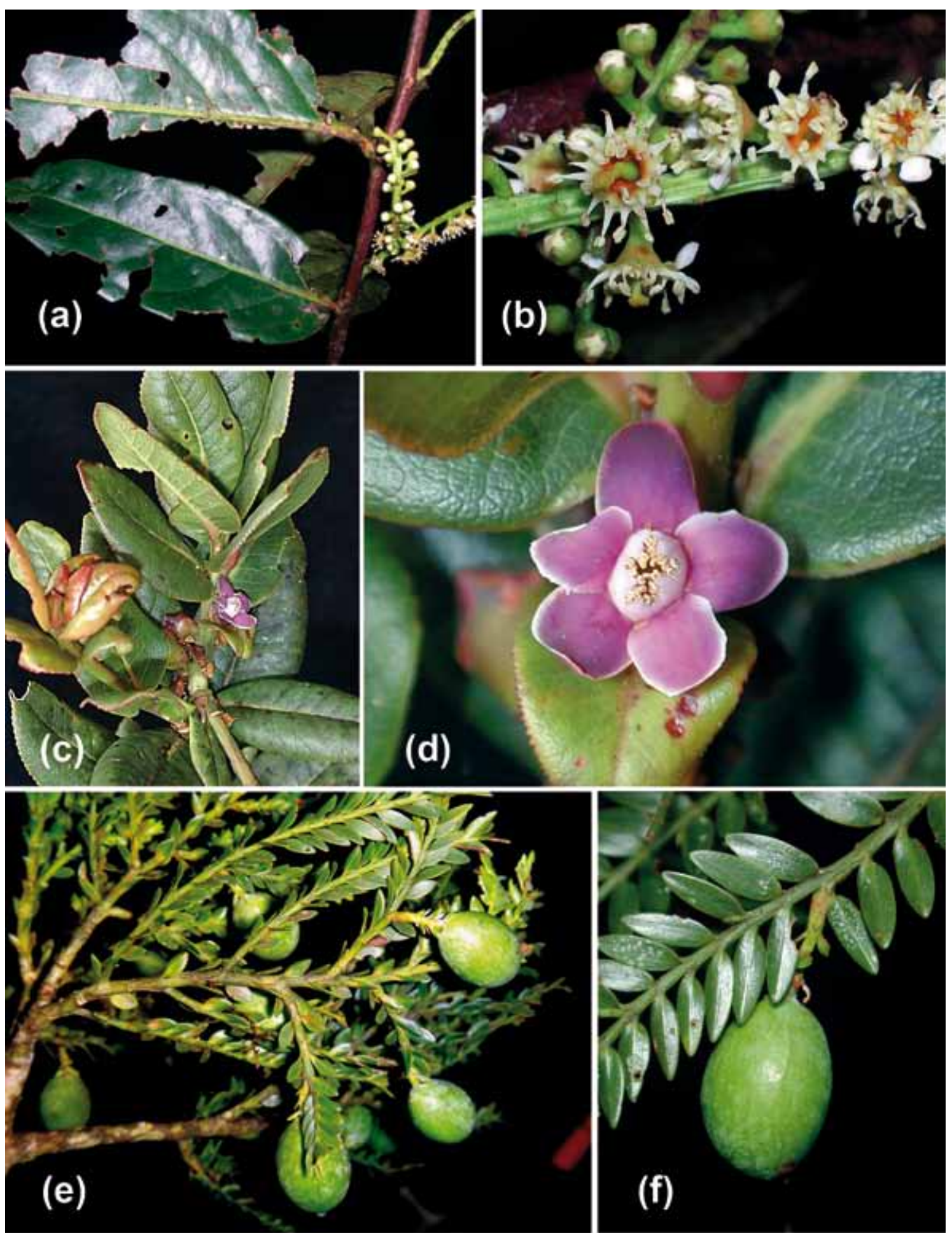

Anexo 3. Prunus integrifolia [W. Farfan, et al. 1191] (a), flor (b). Symplocos psiloclada [W. Farfan, et al. 815] (c) flor (d). Retrophyllum rospigliosii [K. Garcia, et al. 932] (e), frutos (f). Credito de fotos: W. Farfan Rios. 